Research paper

\title{
RTP801 regulates motor cortex synaptic transmission and learning
}

\author{
Leticia Pérez-Sisqués $^{\mathrm{a}, \mathrm{b}, *, 1}$, Núria Martín-Flores ${ }^{\mathrm{a}, \mathrm{b}, 2,1}$, Mercè Masana ${ }^{\mathrm{a}, \mathrm{b}, \mathrm{c}, \mathrm{d}}$, \\ Júlia Solana-Balaguer $^{\mathrm{a}, \mathrm{b}}$, Arnau Llobet ${ }^{\mathrm{a}}$, Joan Romaní-Aumedes ${ }^{\mathrm{a}, \mathrm{b}}$, Mercè Canal ${ }^{\mathrm{a}, \mathrm{b}, 3}$, \\ Genís Campoy-Campos ${ }^{\text {a,b }}$, Esther García-García ${ }^{\mathrm{a}, \mathrm{b}, \mathrm{c}, \mathrm{d}}$, Núria Sánchez-Fernández ${ }^{\mathrm{a}}$, \\ Sara Fernández-García ${ }^{\text {a,b,c,d }}$, James P. Gilbert ${ }^{\mathrm{e}}$, Manuel José Rodríguez ${ }^{\mathrm{a}, \mathrm{b}, \mathrm{c}, \mathrm{d}}$, Heng-Ye Man ${ }^{\mathrm{e}}$, \\ Elena Feinstein $^{\mathrm{f}}$, David L. Williamson ${ }^{\mathrm{g}}$, David Soto ${ }^{\mathrm{a}, \mathrm{b}, \mathrm{c}}$, Xavier Gasull ${ }^{\mathrm{a}, \mathrm{b}, \mathrm{c}}$, Jordi Alberch ${ }^{\mathrm{a}, \mathrm{b}, \mathrm{c}, \mathrm{d}}$, \\ Cristina Malagelada ${ }^{\text {a, b, d,** }}$
}

a Department of Biomedicine, Faculty of Medicine, University of Barcelona, Catalonia, Spain

${ }^{\mathrm{b}}$ Institut de Neurociències, University of Barcelona, 08036, Catalonia, Spain

' IDIBAPS- Institut d'Investigacions BiomèdiquesAugust Pi i Sunyer, Barcelona, 08036, Catalonia, Spain

${ }^{\mathrm{d}}$ Centro de Investigación Biomédica en Red sobre Enfermedades Neurodegenerativas (CIBERNED), Barcelona, 08036, Catalonia, Spain

e Department of Biology, Pharmacology and Experimental Therapeutics, Boston University, Boston, MA 02215, USA

${ }^{\mathrm{f}}$ Quark Pharmaceuticals, Inc. Newark, CA 94560, USA

${ }^{\mathrm{g}}$ Kinesiology Program, School of Behavioral Sciences and Education, Penn State Harrisburg, Middletown, PA 17057, USA

\section{A R T I C L E I N F O}

\section{Keywords:}

GluA1

Motor learning

mTOR

Plasticity

RTP801

\begin{abstract}
A B S T R A C T
Background: RTP801/REDD1 is a stress-regulated protein whose upregulation is necessary and sufficient to trigger neuronal death in in vitro and in vivo models of Parkinson's and Huntington's diseases and is up regulated in compromised neurons in human postmortem brains of both neurodegenerative disorders. Indeed, in both Parkinson's and Huntington's disease mouse models, RTP801 knockdown alleviates motor-learning deficits. Results: We investigated the physiological role of RTP801 in neuronal plasticity and we found RTP801 in rat, mouse and human synapses. The absence of RTP801 enhanced excitatory synaptic transmission in both neuronal cultures and brain slices from RTP801 knock-out (KO) mice. Indeed, RTP801 KO mice showed improved motor learning, which correlated with lower spine density but increased basal filopodia and mushroom spines in the motor cortex layer V. This paralleled with higher levels of synaptosomal GluA1 and TrkB receptors in homogenates derived from KO mice motor cortex, proteins that are associated with synaptic strengthening. Conclusions: Altogether, these results indicate that RTP801 has an important role modulating neuronal plasticity and motor learning. They will help to understand its role in neurodegenerative disorders where RTP801 levels are detrimentally upregulated.
\end{abstract}

\section{Background}

Synaptic plasticity is the ability to fine tune neuronal connectivity and dynamics upon demand, for example in situations in which individuals have to adjust movements in challenging environments. This process is known as motor learning and involves the acquisition of a novel motor skill that, once learned, persists after training period ends (Peters et al., 2017; Sanes and Donoghue, 2000; Xu et al., 2009).

\footnotetext{
Abbreviations: AD, Alzheimer's disease; HD, Huntington's disease; KO, knock out; mTOR, mechanistic target of rapamycin; M1, primary motor cortex; LV, motor

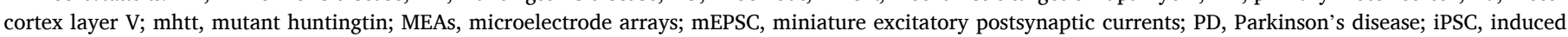

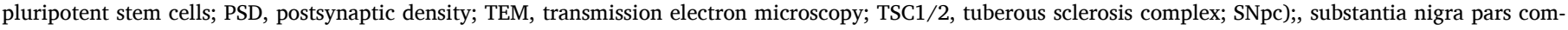
pacta; WT, wild type.

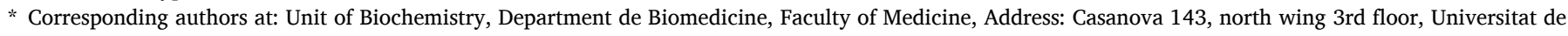
Barcelona, Barcelona 08036, Catalonia, Spain.

E-mail addresses: leticiaperezsisques@gmail.com (L. Pérez-Sisqués), cristina.malagelada@ub.edu (C. Malagelada).

1 These authors contributed equally to this work.

2 Current affiliation: Department of Cell and Developmental Biology, University College London, London, WC1E 6BT, UK.

${ }^{3}$ Current affiliation: HIPRA Headquarters, Amer (Girona) 17170, Catalonia, Spain.
} 
The central hub for motor learning is the motor cortex, an interconnected structure with other brain regions such as the striatum, the thalamus, brainstem or the spinal cord (reviewed in (Shepherd, 2013; Shepherd and Huganir, 2007)). The complex process of acquiring new motor skills induces synaptic plasticity in the motor cortex and requires dendritic spine formation, consolidation and/or elimination, all leading to a necessary synaptic remodeling and strengthening (Fu et al., 2012; Peters et al., 2017; Sanes and Donoghue, 2000; Xu et al., 2009). Pyramidal neurons from the motor cortex and striatal medium spiny neurons (MSNs) predominantly undergo plastic changes along motor learning (Costa et al., 2004; Tjia et al., 2017). Regarding the motor cortex, projection pyramidal neurons from Layer $\mathrm{V}(\mathrm{LV})$ are the main excitatory input to the striatum involved in the corticostriatal pathway (Anderson et al., 2010; Costa et al., 2004; Hintiryan et al., 2016; Shepherd and Huganir, 2007). These plastic changes leading to motor learning involve, at least, increased levels of $\alpha$-amino-3-hydroxy-5-methyl-4isoxazolepropionic acid receptors (AMPAR) at dendritic spines (Kida et al., 2016; Roth et al., 2020). However, the mechanisms by which these events are regulated are not yet clearly elucidated.

In many neurodegenerative diseases, along with neurological and psychiatric symptoms, motor dysfunction is a hallmark of disease progression. Among these disorders, we find Parkinson's disease (PD), Huntington's disease (HD), or amyotrophic lateral sclerosis, (Shepherd, 2013). Motor dysfunction is due, in part, to an impairment in the synaptic plasticity of the circuitries that control movement by interconnecting motor cortex and basal ganglia and the thalamus, and also the cerebellum (Calabresi et al., 2007, 2000; Guo et al., 2015; Xu et al., 2017).

RTP801/REDD1, coded by the DDIT4 gene, is a stress-regulated protein that is sufficient and necessary to induce neuron death (Malagelada et al., 2006; Shoshani et al., 2002). It is elevated in cellular and animal models of PD in response to dopaminergic neurotoxins (Malagelada et al., 2006; Ryu et al., 2005) and is highly up regulated in neuromelanin positive neurons in the substantia nigra pars compacta (SNpc) of both sporadic (Malagelada et al., 2006) and parkin mutant PD patients (Romani-Aumedes et al., 2014). RTP801 induces neuron death by a sequential inactivation of mTOR and the survival kinase Akt (Malagelada et al., 2008) via the tuberous sclerosis complex 1/2 (TSC1/ 2). Regarding HD, RTP801 levels are highly increased in HD human brains, in differentiated neurons derived from induced pluripotent stem cells (iPSC) from HD patients (Martín-Flores et al., 2016) and in striatal synapses from HD mouse models (Martín-Flores et al., 2020). Besides, in neuronal models of the disease, RTP801 mediates mutant huntingtin (mhtt)-induced toxicity (Martin-Flores et al., 2016). Importantly, RTP801 contributes to motor-learning dysfunction in.

both PD and HD since RTP801 knockdown prevents from the appearance of motor learning deficits in the A53T and R6/1 models of these diseases, respectively (Martín-Flores et al., 2020; Zhang et al., 2018).

This suggests that RTP801 deregulation is a common hallmark in neurodegeneration. Indeed, RTP801 coding gene DDIT4 was recently described as one of the top three common deregulated transcripts in postmortem brain samples from PD and HD patients (Labadorf et al., 2018). Furthermore, RTP801 is sufficient to cause neuronal atrophy and depressive-like behavior (Ota et al., 2014) and it has a regulatory role in cortical development, neuronal differentiation (Malagelada et al., 2011) and peripheral nervous system myelination (Noseda et al., 2013). However, its physiological role in synaptic plasticity has not been resolved yet.

For this reason, here we investigated the potential synaptic function of RTP801 in the corticostriatal pathway under physiological conditions. By using cellular and murine models and postmortem human brains and performing behavioral, histological, electrophysiological and biochemical analysis, our results describe the implication of RTP801 in motor learning behavior and synaptic plasticity.

\section{Methods}

\subsection{Animals}

Transgenic RTP801 knock out mouse strain was generated by Lexicon Inc. as described in (Brafman et al., 2004). RTP801 knockout mice were obtained by homozygous pairing. Thus, wild type mice were bred from the RTP801 KO founder strains to obtain a C57B16/129sv background. RTP801 knock out and wild type mice were housed under controlled conditions $\left(22{ }^{\circ} \mathrm{C}, 40-60 \%\right.$ humidity in a 12 -h light/dark cycle) with water and food available ad libitum. All the animals analyzed in this study were 2 months-old adult mice.

For further biochemical analyses, Golgi staining and TEM, mice were euthanized by cervical dislocation and tissue was dissected out. For immunohistochemistry, animals were processed as described elsewhere (Creus-Muncunill et al., 2018). Briefly, animals were anesthetized with $60 \mathrm{mg} / \mathrm{kg}$ dolethal and intracardially perfused with 4\% PFA. Coronal $25 \mu \mathrm{m}$-thick brain sections were obtained with a cryostat.

\subsection{Rat primary cultures}

Rat cortical and hippocampal primary cultures were obtained from embryonic day 18 Sprague-Dawley rats as previously described (Canal et al., 2016). Cells were either transduced with lentiviral particles carrying a control shRNA or a specific shRNA against RTP801 or transfected with lipofectamine 2000 (Thermo Fisher Scientific) with pCMS vectors expressing eGFP (donated by Dr. Lloyd Greene, Columbia University) or eGFP-fused RTP801 protein (Romaní-Aumedes et al., 2014). The sequences to downregulate or overexpress RTP801 were previously described in (Malagelada et al., 2006).

\subsection{Mouse primary cultures}

Mouse primary cortical cultures were obtained from embryonic day 15 mice. Coverslips were coated for $1 \mathrm{~h}$ with $0.1 \mathrm{mg} / \mathrm{ml}$ poly-D-lysine (Merck) and then $3.5 \mathrm{~h}$ with $0.018 \mathrm{mg} / \mathrm{ml}$ laminin (Thermo Fisher Scientific). Briefly, cortices were dissected out and chemically digested with $41.66 \mu \mathrm{M}$ Trypsin for $10 \mathrm{~min}$. Following mechanical digestion, cells were plated on coverslips at a density of $25.000 \mathrm{cells} / \mathrm{cm}^{2}$ and maintained in Neurobasal-A medium supplemented with B27, GlutaMAX (all from Gibco), $33.3 \mathrm{mM}$ Glucose and 1\% penicillin-streptomycin (Sigma) in a $5 \% \mathrm{CO}_{2}$ atmosphere and $37{ }^{\circ} \mathrm{C}$.

\subsection{Crude synaptosomal fractionation}

Tissue (rat, mice or postmortem human brains) or cultured cells were homogenized in Krebs-Ringer buffer (125 mM NaCl, $1.2 \mathrm{mM} \mathrm{KCl}, 22 \mathrm{mM}$ NaHCO3, $1 \mathrm{mM}$ NaH2PO4, $1.2 \mathrm{mM} \mathrm{MgSO}$, $1.2 \mathrm{mM} \mathrm{CaCl}$, $10 \mathrm{mM}$ Glucose, 0.32 M Sucrose; pH 7.4). For samples in Fig. 7, mice were sacrificed one week after behavioral testing. Initial lysate was first centrifuged at $1.000 \mathrm{~g}$ for $10 \mathrm{~min}$. Supernatant (homogenate) was centrifuged for $20 \mathrm{~min}$ at $16.000 \mathrm{~g}$ to obtain the cytosolic fraction (supernatant) and the crude synaptosomal fraction (pellet), that was resuspended in Krebs-Ringer buffer.

\subsection{Western blotting}

Samples were resolved in NuPAGETMNovex ${ }^{\mathrm{TM}}$ polyacrylamide gels and proteins were transferred to nitrocellulose membranes with the iBlot system (all from Thermo Fisher Scientific). Indicated primary antibodies were incubated overnight at $4{ }^{\circ} \mathrm{C}$ diluted in Tris-buffered saline containing $0.1 \%$ Tween-20 and 5\% BSA. Secondary antibodies (Thermo Fisher Scientific) were diluted in TBS-Tween with non-fat dry $5 \%$ milk (Bio-rad) for $1 \mathrm{~h}$. Proteins were detected with Supersignal ${ }^{\mathrm{TM}}$ West Pico Plus chemiluminiscent substrate (Thermo Fisher Scientific) and images were acquired with ChemiDoc ${ }^{\mathrm{TM}}$ (Bio-Rad). The following antibodies 
were used: RTP801 (1:500, Proteintech), HRP-conjugated anti-beta actin (1:100.000; Sigma), PSD-95 (1:1000; Thermo Fisher Scientific), SV2a and GFP (1:1000; Santa Cruz Biotechnology), GluA1, GluA2, Stargazin (1:1000; Merck Millipore), GluN2B (1:1000; Cell Signaling Technology), BDNF (1:1000; Icosagen) and TrkB (1:1000; BD Biosciences).

\subsection{Immunofluorescence}

Cells were fixed in 4\% PFA and permeabilized with $0.25 \%$ Triton-X. Blocking and antibody incubation was performed with Superblock (Thermo Fisher Scientific). Primary antibodies were incubated over night at $4{ }^{\circ} \mathrm{C}$ and secondary antibodies for $2 \mathrm{~h}$ at room temperature. For mouse brain tissue immunofluorescence, sections were washed with PBS and incubated for $30 \mathrm{~min}$ in $\mathrm{NH}_{4} \mathrm{Cl}$. Next, sections were blocked with $0.3 \%$ Triton-100 10\% NGS in PBS for $2 \mathrm{~h}$ prior incubation with the primary antibodies diluted in blocking solution overnight at $4{ }^{\circ} \mathrm{C}$. Later, sections were washed and incubated for $2 \mathrm{~h}$ with the secondary antibodies. Slices were then washed with PBS. Both cells and tissue samples were mounted with Prolong Gold antifade mountant (Thermo Fisher Scientific). The following antibodies were used: GFP (1:500), SV2a (1:100) (both from Santa Cruz Biotechnology), PSD-95 (1:50; Thermo Fisher Scientific), GluA1 (1:250-1:500; Merck Millipore) and RTP801 (1:100; Proteintech). AlexaFluor-488 or -555 secondary antibodies (1:500) and Hoechst33342 (1:5000) were from Thermo Fisher Scientific. Images were obtained with a Leica LCS SL or a Zeiss LSM880 confocal microscopes with a $1024 \times 1024$ pixel resolution and a $63 \times$ magnification and were analyzed with ImageJ. For in vitro experiments in cortical neurons, at least 25 dendrites per group from three independent experiments were analyzed. For in vitro experiments in hippocampal neurons, at least 12 neurons per group were analyzed from three independent experiments. For double-labeled GluA1-PSD-95-positive clusters in brain slices, images were acquired with $4 \times$ digital zoom $(33.74 \times 33.74 \mu \mathrm{m})$. For each mouse three representative images from two different coronal sections were analyzed. Colocalization was considered when there was at least one common pixel between GluA1 and PSD-95 detected puncta.

\subsection{Nissl staining}

Slices were stained for $45 \mathrm{~min}$ with $0.2 \mathrm{mg} / \mathrm{ml}$ Cresyl violet (Sigma) in a $0.1 \mathrm{M}$ acetic acid $0.1 \mathrm{M}$ sodium acetate solution. Next, slices were washed in distilled water and then dehydrated with ethanol (70, 95, $100 \%, 5$ min each), washed with xylol and mounted with DPX media. Images were obtained with a $10 \times$ magnification with a Zeiss Axiolab.

\subsection{Behavioral assessment}

\subsubsection{Footprint test}

Mice's fore and hindlimbs were painted in blue and red, respectively, with non-toxic ink. Animal's gait was then recorded letting them walk through a tunnel on white paper $(10 \mathrm{~cm}$ wide, $40 \mathrm{~cm}$ long). The test was performed three times on the same day. In each trial three consecutive steps were measured for each parameter (stride, sway, stance, overlap).

Open field test: mice were placed in a $40 \times 40 \times 40 \mathrm{~cm}$ arena. The center area was considered as the central squared $20 \times 20 \mathrm{~cm}$ space. Light intensity was $24 \mathrm{~lx}$ though-out the periphery and $29 \mathrm{~lx}$ in the center. Mice's movement was tracked and recorded for $10 \mathrm{~min}$ using SMART 3.0 Software (Panlab). Other parameters related to anxiety-like behaviors, like number of groomings, rearings and defecations were also monitored.

\subsubsection{Accelerating rotarod}

One day after the Open field test mice were subjected to the Accelerating rotarod test. Mice were placed on a $3 \mathrm{~cm}$ rod with an increasing speed from 4 to $40 \mathrm{rpm}$ over $5 \mathrm{~min}$. Latency to fall was recorded as the time mice spent in the rod before falling. Accelerating rotarod test was performed for 4 days, 4 trials per day. Trials in the same day were separated by $1 \mathrm{~h}$.

\subsubsection{Clasping behavior}

Hindlimb clasping was measured by picking up mice at the base of the tail. In order to classify this phenotype we used the scale described in (Guyenet et al., 2010) with minor modifications: 0 means no hind paw retraction, 1, one hindlimb retracted, 2, both hindlimbs partially retracted, and 3 when the 2 hindlimbs were totally retracted.

\subsection{Golgi staining and spine density and morphology analyses}

Golgi-Cox impregnation was performed with fresh brain hemispheres from, mice sacrificed one week after behavioral testing with FD Rapid GolgiStain ${ }^{\mathrm{TM}}$ kit (FD Neurotechnologies) following manufacturer's instructions. $100 \mu \mathrm{m}$ slices were obtained with a Leica vibratome and mounted on gelatin-coated slides before final staining.

For spine density analyses only pyramidal neurons from layer $\mathrm{V}$ in the primary motor cortex or medium spiny neurons (MSNs) from the dorsolateral striatum were taken into account. Spine density was quantified in dendritic segments of at least $10 \mu \mathrm{m}$ and 30 different secondary/tertiary dendrites per animal were analyzed. Analyzed dendrites were $50 \%$ apical, $50 \%$ basal.

Spine morphology analyses were performed in motor cortex layer $\mathrm{V}$ pyramidal neurons. Spines in 5 apical and 5 basal secondary/tertiary dendrites were analyzed for each animal ( $6 \mathrm{WT}$ and $4 \mathrm{KO}$ ), in segments of at least $10 \mu \mathrm{m}$ long. A total of 100-125 apical and 100-125 basal spines were analyzed per animal. Branched, filopodia and stubby spines were visually categorized. For headed spines, head area was measured in all headed spines and thin/mushroom classification was performed depending on the mean head area for each genotype (spines with head area greater than the mean were considered as mushroom spines and smaller ones were categorized as thin spines). In spine density and morphology analyses, animal genotype was blind for the experimenter.

\subsection{Transmission electron microscopy}

2 months old RTP801 knock out $(n=4)$ and wild type mice $(n=4)$ were sacrificed one week after behavioral testing and motor cortex was dissected from coronal sections. From these sections, the lower half of the motor cortex, including Layer V and VI, was isolated and fixed overnight in $2 \%$ glutaraldehyde $2 \%$ paraformaldehyde in $0.12 \mathrm{M}$ phosphate buffer. After fixation, tissue was processed and analyzed as previously described in (Bosch et al., 2016). Electron micrographs were randomly taken at $25.000 \times$ with a TEM JEOL J1010 (tungsten filament), with a CCD Orius (Gatan) and software Digital Micrograph (Gatan). Spine density, pre/postsynaptic area and postsynaptic density area, length and thickness were determined ( $n=45-50$ images for each animal) with ImageJ software. In all TEM analyses, animal genotype was blind for the experimenter.

\subsection{Electrophysiology}

\subsubsection{Rat neuronal cultures}

Miniature excitatory postsynaptic currents (mEPSCs) were measured in rat primary hippocampal neurons plated on glass coverslips as previously described (Gilbert et al., 2016).

\subsubsection{Mouse cortical cultures}

Electrophysiological recordings of cultured cortical pyramidal neurons -chosen in basis of their characteristic pyramidal morphologywere performed at $14 \mathrm{DIV}$. Whole-cell patch-clamp currents were recorded at room temperature $\left(25-26{ }^{\circ} \mathrm{C}\right)$ in extracellular solution containing (in $\mathrm{mM}$ ): $130 \mathrm{NaCl}, 3.5 \mathrm{KCl}, 10$ HEPES, 15 glucose and 2 $\mathrm{CaCl}_{2}$ (pH 7.4; osmolarity $305 \mathrm{mOsm} / \mathrm{Kg}$ with sorbitol). AMPAR- 
mediated miniature excitatory postsynaptic currents (mEPSCs) were isolated adding to the extracellular solution $1 \mu \mathrm{M}$ tetrodotoxin to block evoked synaptic transmission, $100 \mu \mathrm{M}$ picrotoxin to block $\mathrm{GABA}_{\mathrm{A}}$ receptors and $50 \mu \mathrm{M}$ APV to block NMDA receptors. Recording electrodes were fabricated from borosilicate glass with a final resistance of 4-5 M $\Omega$ and filled with an internal solution containing (in mM): $120 \mathrm{~K}$-Gluconate, $16 \mathrm{KCl}, 8 \mathrm{NaCl}, 10$ HEPES, 0.2 ethylene glycol tetraacetic acid (EGTA), 2 MgATP, $0.3 \mathrm{Na}_{2} \mathrm{GTP}$ (pH 7.2; osmolarity 291 with sorbitol). Recordings were acquired at a sampling rate of $5 \mathrm{KHz}$ and were filtered at $2 \mathrm{~Hz}$. Miniature events were detected and analyzed with the WaveMetrics Igor Pro open-source software package Neuromatic (Rothman and Silver, 2018). Frequency was determined by dividing the number of detected events by the recorded time (in seconds).

\subsection{Electrophysiological field recordings}

Two-month old (female and male) mouse brain sagittal sections were obtained on a vibratome (Microm HM $650 \mathrm{~V}$, Thermo Scientific, Waltham, MA, USA) at $350 \mu \mathrm{m}$ thickness in oxygenated $\left(95 \% \mathrm{O}_{2}, 5 \% \mathrm{CO}_{2}\right)$ ice-cold aCSF and then transferred to a oxygenated $32{ }^{\circ} \mathrm{C}$ recovery solution for $15 \mathrm{~min}$ as previously described (Choi et al., 2019). Then, slices were transferred to oxygenated aCSF at room temperature and left for at least $1 \mathrm{~h}$ before electrophysiological field recording. Following recovery, mouse $350 \mu \mathrm{m}$ thick brain slices were placed in a multi electrode array (MEA) recording dish and fully submerged in oxygenated aCSF at $37^{\circ} \mathrm{C}$. Electrophysiological data were recorded with a MEA set-up from Multi Channel Systems MCS GmbH (Reutlingen, Germany) composed of a 60 channels USB-MEA60-inv system. Experiments were carried out with 60MEA200/30iR-ITO MEA dishes consisting of 60 planar electrodes (30 $\mu \mathrm{m}$ diameter) arranged in an $8 \times 8$ array and placed in the motor cortex slice surface. Raw traces were recorded for $5 \mathrm{~min}$ from 58 electrodes simultaneously, sampled at $5 \mathrm{kHz}$. Raw data were high-pass filtered with a 200-Hz Butterworth 2nd order filter, the noise level calculated by the standard deviation of the recorded signal on each electrode and spikes were identified as currents with a negative amplitude larger than $-30 \mathrm{mV}$ and slope values between 0.2 and 1 . To quantify burst activity in spike-trains we applied the MaxInterval Method (Legendy and Salcman, 1985) with the following parameter values: maximum beginning ISI, $200 \mathrm{~ms}$; maximum end ISI, $200 \mathrm{~ms}$; minimum interburst interval, $20 \mathrm{~ms}$, minimum burst duration $20 \mathrm{~ms}$; minimum number of spikes in a burst, 5. Software for recording and signal processing was MC Rack from Multi Channel Systems. Using a digital camera during recording assessed the position of the brain slices on the electrode field to analyze information from electrodes specifically positioned on cortical layer V (Fig. $2 \mathrm{H}$ ).

\subsection{Experimental design and statistical analyses}

Graphs show results reported as mean \pm SEM. Data was assessed for normality using D'Agostino-Pearson, Shapiro-Wilk or KolmogorovSmirnov. Statistical analyses were performed using unpaired, twotailed Student's $t$-test for normally distributed data, Mann-Whitney test for non-parametric data and Two-way ANOVA followed by Bonferroni's post-hoc tests to compare multiple groups, as appropriate and indicated in the figure legends. Values of $P<0.05$ were considered as statistically significant.

\section{Results}

\subsection{RTP801 is localized in the synapses of murine and human samples and modulates synaptic transmission in vitro}

We first explored whether RTP801 was localized in synapses and whether it was involved in synaptic function, connectivity and transmission. Hence, we first isolated cortical and striatal crude synaptosomes from adult postmortem human brain, adult rat and mouse brains and from cultured rat cortical neurons. In all samples we observed the presence of RTP801 or its enrichment in crude isolated synaptic terminals in comparison to the initial homogenates (Fig. $1 \mathrm{~A}$ ), corroborating our own previous results (Martín-Flores et al., 2020). Interestingly, in cultured cortical neurons, we observed that RTP801 was expressed in the soma, dendrites and dendritic spines (Fig. 1 B).

We next investigated whether RTP801 depletion affected spine density and synaptic transmission. For this, we knocked down the expression of RTP801 in cortical primary cultures at 14DIV, using lentivirus expressing a specific shRNA for RTP801 or scramble shRNA as control. We observed that RTP801 silencing induced a significant decrease in spine density relative to the scramble shRNA transduced neurons (Fig. 1 C). We next analyzed whether RTP801 expression abrogation affected synapse function by evaluating the frequency and the amplitude of mEPSCs of cortical cultures derived from WT and RTP801 KO mice. Interestingly, in the complete absence of RTP801 expression using cultured cortical neurons from RTP801 KO mice, we observed that both the amplitude (Fig. 1 D1, D2 \& D.3) and frequency (Fig. 1 D1, D2 \& D.4) of mEPSCs were higher than the ones registered in WT cortical sister cultures.

We corroborated our in vitro results using cultured hippocampal neurons, a well characterized plasticity model. In line with previous results, we found that RTP801 colocalized with PSD-95, an excitatory postsynaptic scaffold protein, but not with the presynaptic marker SV2A, indicating that RTP801 is localized in the postsynaptic compartment (Fig. S1 A-B). Moreover, ectopic RTP801 expression attenuated the amplitude of mEPSCs without affecting the frequency, along with a decrease of PSD-95 and AMPAR receptor subunit GluA1 puncta intensity (Fig. S1 C-E).

\subsection{Synaptic and behavioral characterization of RTP801 KO mice brains}

Previous data pointed out that the total abrogation of RTP801 expression did not influence significantly either the brain structure or the basal behavior of the RTP801 KO mice in comparison to WT animals (Brafman et al., 2004; Ota et al., 2014). However, we previously demonstrated that RTP801 regulated the timing of cortical neurogenesis and neuron differentiation/migration (Malagelada et al., 2011) using in utero electroporation techniques. For this reason, to validate the use of the RTP801 KO mouse to study its putative synaptic role, we characterized its brain morphology in comparison to WT animals. We first confirmed the lack of RTP801 expression in the KO animals in motor cortex homogenates (Fig. 2 A). Macroscopically, although there were no differences in the mice body weight between genotypes (Fig. 2 B), we observed that $\mathrm{KO}$ animals presented a decreased brain weight (Fig. 2 C). However, internal structural organization did not present major alterations either in cortical layers, hippocampus or even in the striatum, as judged by Nissl staining (Fig. 2 D). Primary motor cortex (M1) layer thickness did not differ either between genotypes (Fig. 2 E) but RTP801 KO mice showed an expected decreased cell density in the M1 LV (Fig. 2 F).

We next investigated whether cortical spine density was affected in the adult brain of RTP801 KO mice using Golgi-Cox staining. Analyses were performed in the M1 LV pyramidal neurons, the main excitatory and direct projection to the ipsi- and contralateral striatum in the corticostriatal pathway (Anderson et al., 2010; Hintiryan et al., 2016; Shepherd, 2013; Xu et al., 2009). As previously seen by knocking down RTP801 in cortical cultured neurons (see Fig. 1 C), we observed a reduction in the density of spines in LV neurons in naive RTP801 KO mice compared to WT animals (Fig. $2 \mathrm{G}$ ).

Next, we assessed whether RTP801 modulates synaptic transmission in cortical brain slices from naïve WT and KO animals. We thus measured neuronal spike rate and bursting in M1 LV using multielectrode array (MEA) (Fig. $2 \mathrm{H}$ ). We found an increased spike rate in the M1 LV of KO animals when compared with WT (Fig. 2 I.1), with no differences between male and female animals (Fig. S2 A). Analysis of spike- 
A
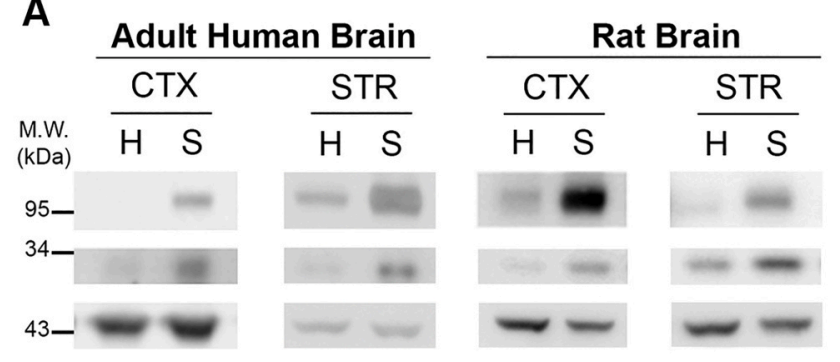

Rat \begin{tabular}{llll} 
cortical & PC12 & \multicolumn{2}{c}{ Mouse CTX } \\
& cells & WT & KO
\end{tabular} H S $\overline{\mathrm{H} \mathrm{S}} \overline{\mathrm{H} \mathrm{S}}$

PSD-95
*RTP801 $-$
Actin

B
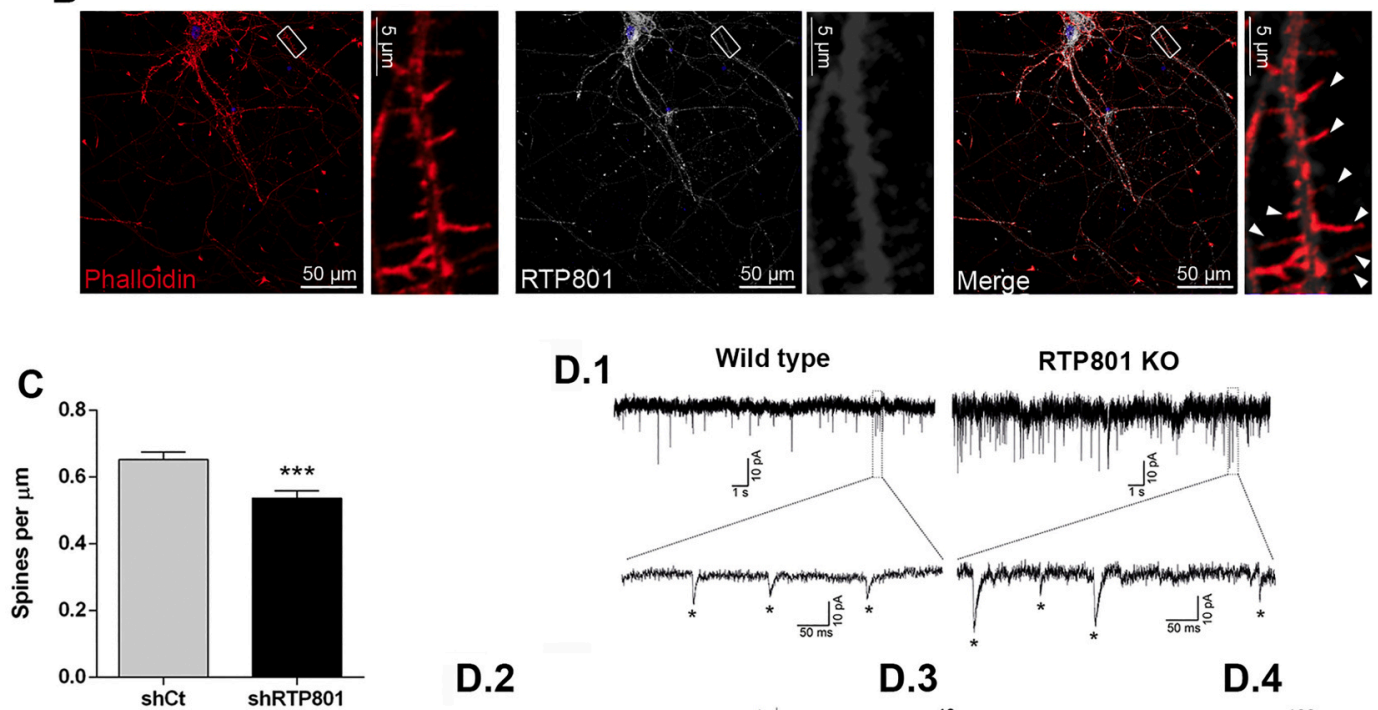

D. 1

Wild type

RTP801 KO

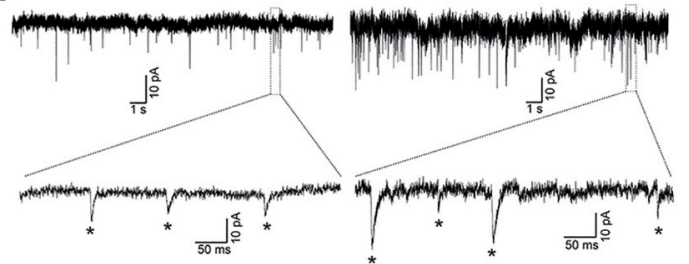

D.2

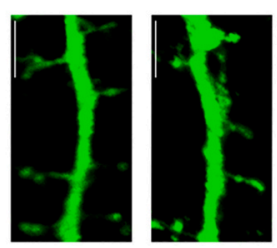

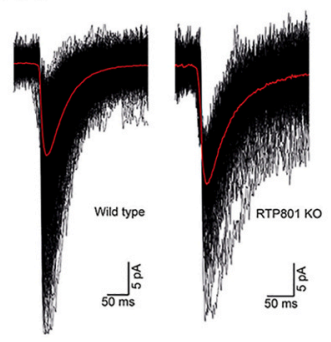

D. 3

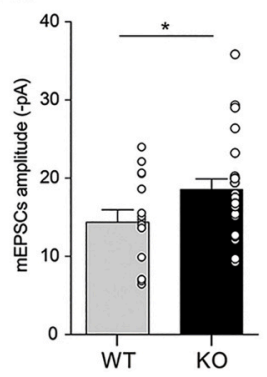

D. 4

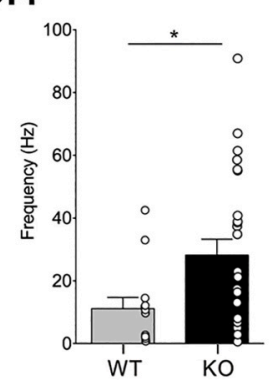

Fig. 1. RTP801 is present at the synapse and modulates neuronal transmission. A. RTP801 protein is found in the synaptic compartment. Homogenate (H) and crude synaptic fraction (S) were obtained from human (6 h of postmortem interval, 76 years, male, with no neuropathological evidences) postmortem cortex (CTX) and putamen (STR), adult rat cortex (CTX) and striatum (STR), primary cortical cultures at 14 DIVs and cortex from both 2-months old WT and RTP801 KO mice. Whole cell lysate (L) from NGF-differentiated PC12 cells was added as a positive control to detect RTP801 in mouse brains. Samples were analyzed by western blot and proved against RTP801 (specific band pointed out by *), postsynaptic protein PSD-95 and actin as a loading control. B. RTP801 is present ubiquitously in neurons, including at the synapses. Primary rat cortical cultures were fixed at 14DIVs and stained for RTP801 (grey). Phalloidin (red) was used to visualize actin cytoskeleton. Nuclei were stained with Hoechst33342 (in blue). White arrows point F-actin-labeled dendritic spines colocalizing with endogenous RTP801 staining. C. RTP801 knockdown reduces spine density in cultured cortical neurons. Primary rat cortical neurons were transduced with lentiviral particles carrying a GFP-tagged control shRNA or an shRNA against RTP801. 4 days later (14 DIVs), cells were fixed and analyzed by immunofluorescence against GFP (green). Scale bar, $5 \mu m$. D. Abrogation of RTP801 expression modulates synaptic plasticity in vitro. D.1. Representative $20 \mathrm{~s}$ whole-cell recording of mEPSCs at a membrane voltage of $-70 \mathrm{mV}$ from WT or RTP801 KO mice cultured cortical pyramidal neurons (14 DIV). A magnification ( $0.5 \mathrm{~s})$ for both traces is shown below where asterisks denote the detected events. D.2. Example of averaged mEPSCs (red lines) superimposed on the individual mEPSCs (in black) from a wild type (average of 962 events) and RTP801 knockout (average of 236 events) culture. D.3. RTP801 KO recordings show differences in mEPSCs mean amplitude. D.4. The frequency of detected events in RTP801 KO neurons was statistically increased compared with WT. mEPSCs frequencies were obtained from same recordings shown in D.3. All data is presented as mean \pm SEM from the recordings performed in 15 WT neurons and $25 \mathrm{KO}$ neurons from at least six independent neuronal cultures. Statistical analyses for spine density and mEPSC amplitude were performed with Student's $t$-test, ${ }^{*} P<0.05$, ${ }^{* * *} \mathrm{P} 0.001 v$ s. shCt/WT and with Mann-Whitney test for mEPSC frequency, ${ }^{*} \mathrm{P}<0.05 v s$. WT.

train patterns showed a higher burst rate and proportion of spikes included in bursts in KO primary motor cortex slices when compared with WT (Fig. 2 I.2-I.3). We found no other differences in the burst parameters analyzed (Fig. S2 B-D). These results support the hypothesis that neuronal excitability is increased in LV motor cortex in KO mice as an attempt to compensate the decreased number of synaptic spines. Importantly, this does not have any epileptogenic effect.

To study whether synaptic structural and functional changes in
RTP801 KO mice correlated with behavioral alterations, we next investigated whether the lack of RTP801 affected coordination, locomotion and motor learning. We first tested WT and KO mice for hindlimb clasping, a marker of disease progression in a number of mouse models of neurodegeneration, including HD (Chou et al., 2008; Mangiarini et al., 1996). We observed that RTP801 KO male mice displayed a clasping phenotype, not present in male WT mice. The tendency in females was similar but not significant (Fig. S3 A). We next explored 


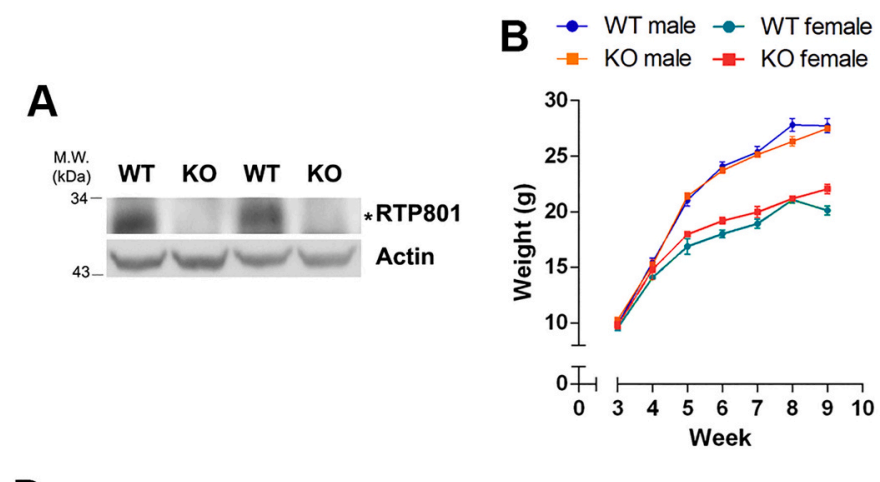

D
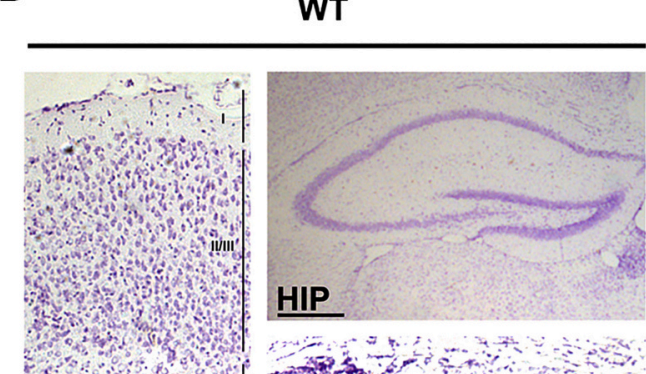

$-5$
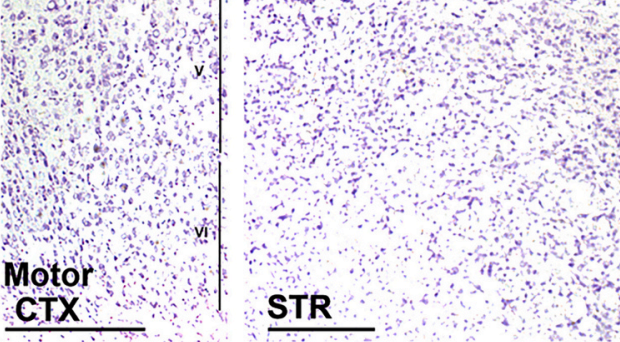

C

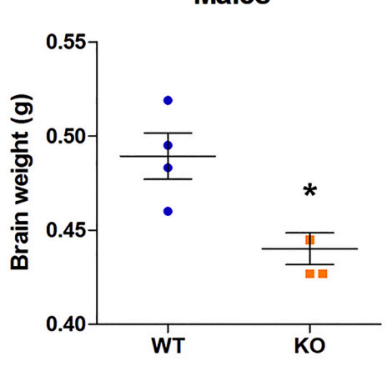

Ko
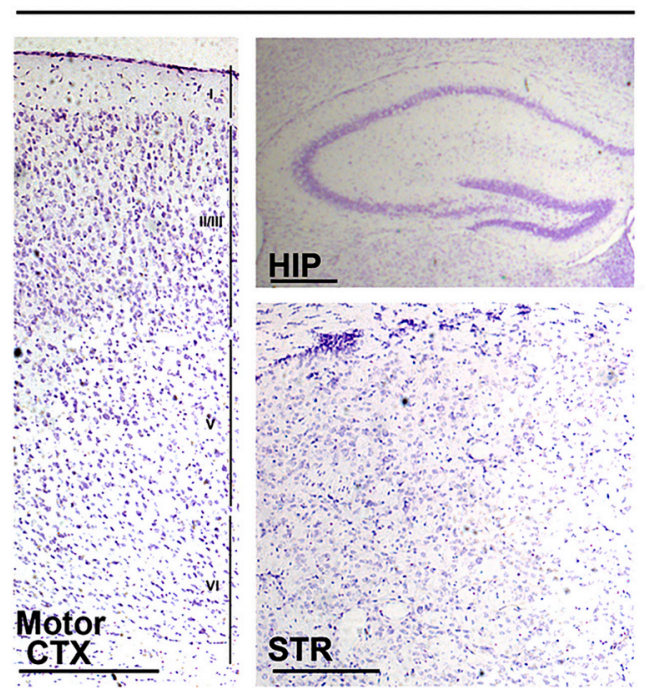

E

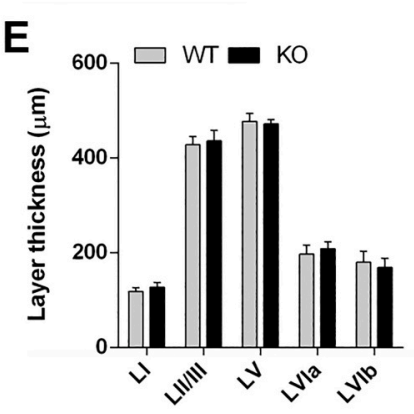

F
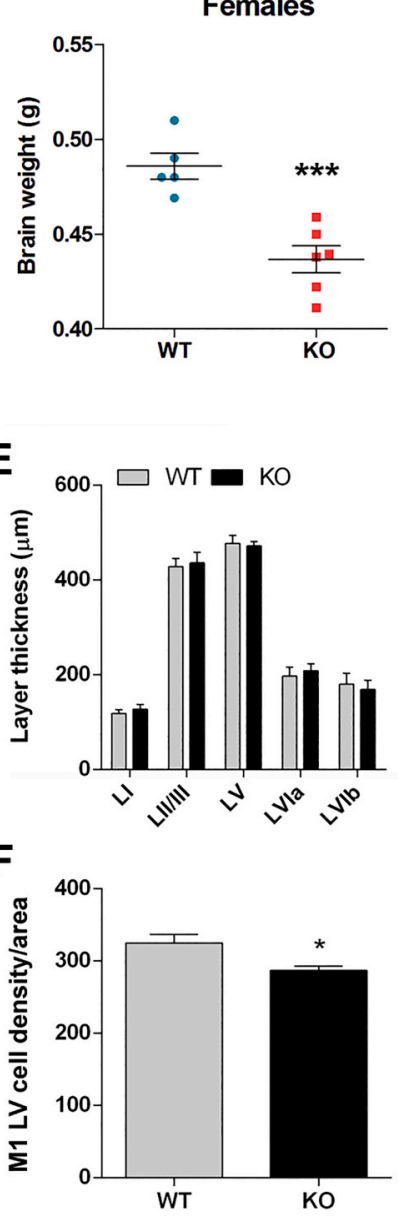

G

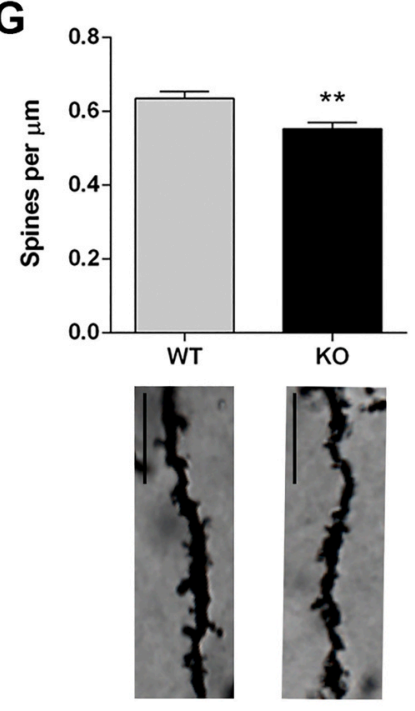

H

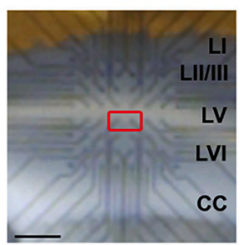

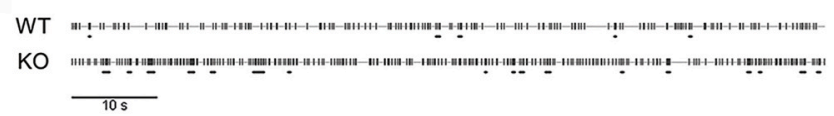

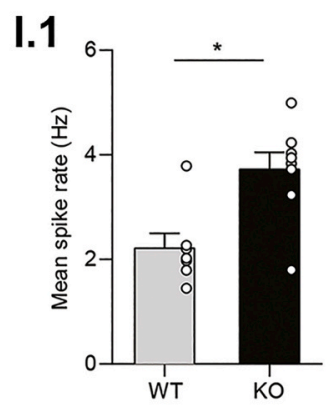


Fig. 2. RTP801 KO mice show decreased brain size, decreased cortical spine density and enhanced synaptic transmission.

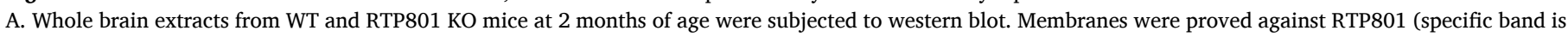

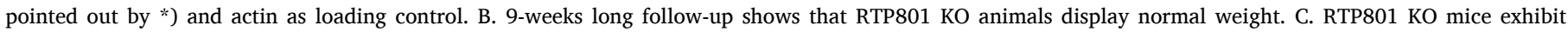

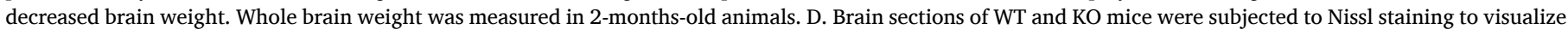

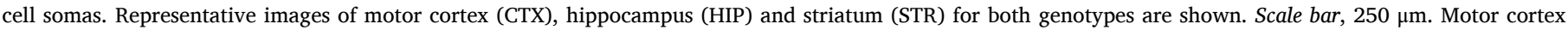

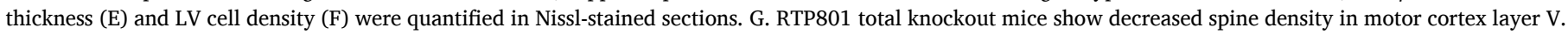

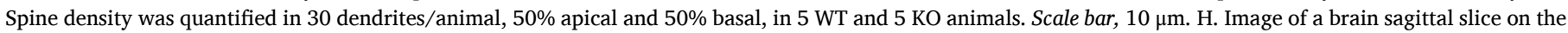

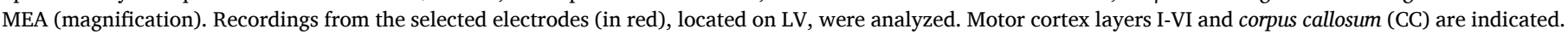

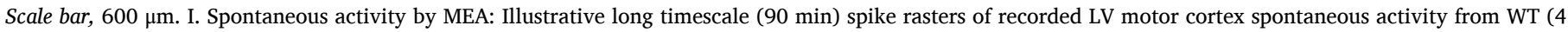

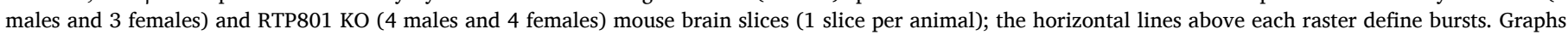

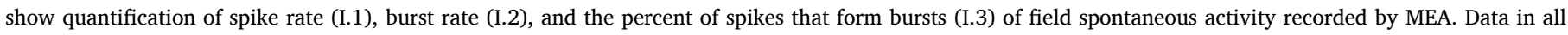

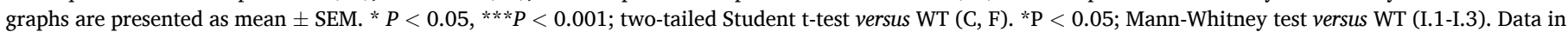
(B, E) was analyzed by two-way ANOVA.

whether gait, as a measure of coordination and muscle function, was affected in RTP801 KO mice. These animals showed a decrease in the length of the stride, stance, sway and the overlap (Fig. 3 A-B and Fig. S3 B), suggesting gait impairment in the KO animals. We next examined whether general locomotor activity was altered using the Open Field test. Despite gait impairment, we did not find any differences in the total distance traveled in the RTP801 KO mice relative to WT (Fig. $3 \mathrm{C}$ ). We did not find differences in the distance traveled in the center or the time spent in the center, suggesting that RTP801 KO mice do not exhibit anxiety-like behavior. Regarding other general exploratory and stereotypic behavior, we did not find any differences in grooming or wall and vertical rearing, either (Fig. S3 C).

To evaluate motor skill learning, we trained the WT and RTP801 KO animals in the accelerating rotarod. Both female and male KO mice showed the same trend to improve motor learning in this behavioral paradigm (Fig. S3 D). Together, RTP801 KO mice significantly improved performance in this task compared to WT animals (Genotype effect, ** $P=0.0058$ ) (Fig. 3 D). Moreover, although we did not observe any differences in the early learning phase (day 1 ), we did find a significantly higher learning rate in the KO animals at day 2 that is sustained at day 3 (Fig. 3 D). In addition, we confirmed that the training associated with learning a motor task did not modulate the levels of RTP801 in WT mice (Fig. S4). Hence, these results indicate that motor learning does not modulate RTP801 levels but RTP801 is involved in motor learning acquisition.

\subsection{RTP801 modulates spine density and structure in the primary motor cortex of trained animals}

We next investigated whether the improvement in motor learning in the RTP801 KO mice affected differentially spine density and structure. Hence, since motor learning plasticity involves projections from the motor cortex to the dorsal striatum, we explored spine density and morphology in pyramidal neurons from the M1 LV and in medium spiny neurons (MSNs) from the dorsal striatum, one week after finishing the accelerating rotarod test (Fig. 4 A). Similar to non-trained naïve RTP801 KO mice, trained RTP801 KO mice showed a decrease in the density of spines in LV pyramidal neurons (Fig. 4 B), specifically in their basal dendrites. Interestingly, spine density of either cortical LV apical dendrites or dendrites in striatal MSNs did not change (Fig. 4 C-D).

Based on these results, we investigated differences in spine morphology in the M1 LV pyramidal neurons that could explain the increased motor learning in the KO mice. Indeed, RTP801 KO animals displayed more filopodia but less branched spines (Fig. 5 A and Fig. S5). In line with this, when related with the total number of headed spines, we observed a higher percentage of mushroom spines in the basal dendrites of KO animals (Fig. 5 C.1, C.2). Moreover, their head area was also increased. On the contrary, no differences were found in either the percentage or head area of thin spines (Fig. 5 B.1, B.2).

We next asked whether this evidence in LV pyramidal neurons based on Golgi-Cox staining could be supported ultra-structurally by Transmission Electron Microscopy (TEM). Interestingly, we observed that KO mice synapses had bigger postsynaptic area (around 10\%) (Fig. 6 A) along with a wider PSD area, length and thickness (around 5\%, each) (Fig. 6 B). Interestingly KO mice exhibited a higher percentage of contacts containing mitochondria, mostly at the presynaptic compartment although the postsynaptic compartment showed a similar tendency (Fig. 6 C). We did not find significant differences in the percentage of presynapses with more than one post-synapse, postsynapses with more than one presynapse or postsynapses with spine apparatus (Fig. S6 A-C). Altogether, these results support the idea that although the KO mice have a decreased number of spines in the motor cortex LV, they displayed a more efficient synaptic structure.

\subsection{The lack of RTP801 elevates GluA1 AMPAR post-synaptically}

In line with the reduction in spine density in neurons from motor cortex LV in the RTP801 KO mice (Fig. 4B), biochemical analysis of KO motor cortex crude synaptic fractions confirmed a decrease in PSD-95 (Fig. 7 A) but an specific enrichment of synaptic GluA1 (Fig. 7 B), a crucial AMPAR subunit that has been described to be a key mediator in the acquisition of new motor skills (Kida et al., 2016; Roth et al., 2020). On the other hand, GluA2 AMPAR subunit, the prototypical auxiliary subunit of AMPARs stargazin or the $N$-methyl-D-aspartate receptor (NMDAR) subunit GluN2B did not change in KO mice in comparison to WT (Fig. 7 B-C). Interestingly, although we did not observe differences in the levels of BDNF between genotypes (Fig. S7 A-D), we did observe that levels of TrkB were elevated in total homogenates in the RTP801 KO motor cortex (Fig. 7 D), supporting the idea of a synaptic strengthening.

Furthermore, we also investigated whether the GABAergic neurons were affected in the cortex of the KO mice and we did not find any differences in the levels of the vesicular GABA transporter (VGAT) (McIntire et al., 1997), or the levels of calbindin and parvalbumin by WB. We did not observe either any changes in the levels of the inhibitory postsynaptic scaffolding marker Gephyrin (Fig. S7 E-I). Therefore, GABAergic neurons in the motor cortex in the absence of RTP801 seem to be spared.

By immunostaining WT and KO sections against PSD95 and GluA1 postsynaptic markers, we confirmed these initial biochemical observations specifically in M1 layer V. Indeed, the number of PSD-95 and GluA1 puncta diminished in the KO animals (Fig. 7 E, H) although the area and the intensity of the GluA1 dots were increased (Fig. 7 F-G). Area and intensity of PSD-95 positive dots showed a non-significant increased tendency, as well (Fig. 7 F-G). Altogether, these results suggest a novel synaptic role for RTP801 modulating synaptic strength and motor learning in the motor cortex (Fig. 8).

\section{Discussion}

Here, we show a novel role for RTP801 in the modulation of synaptic plasticity and motor learning. The lack of RTP801 in mice resulted in decreased spine density and enhanced synaptic transmission in the primary motor cortex together with a better performance in the accelerating rotarod but altered gait and clasping. This improvement in motor 
A

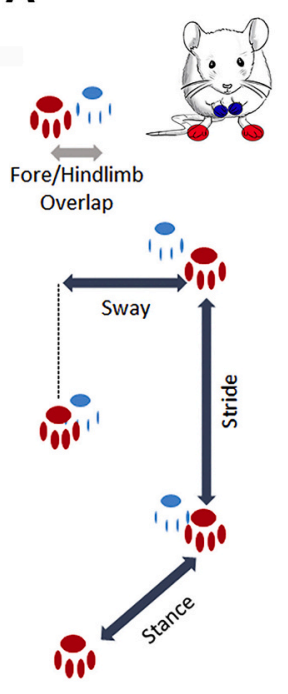

B

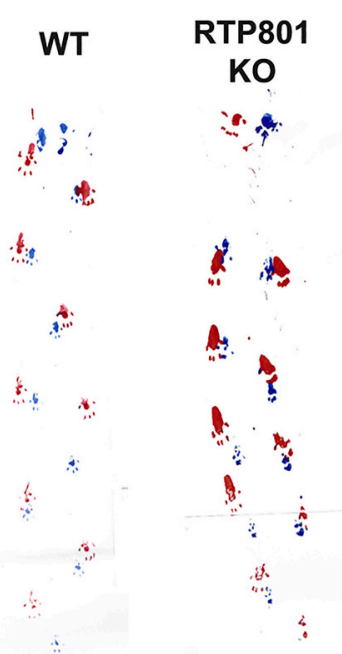

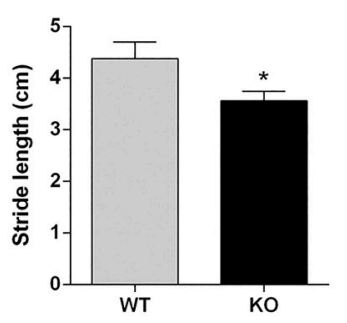
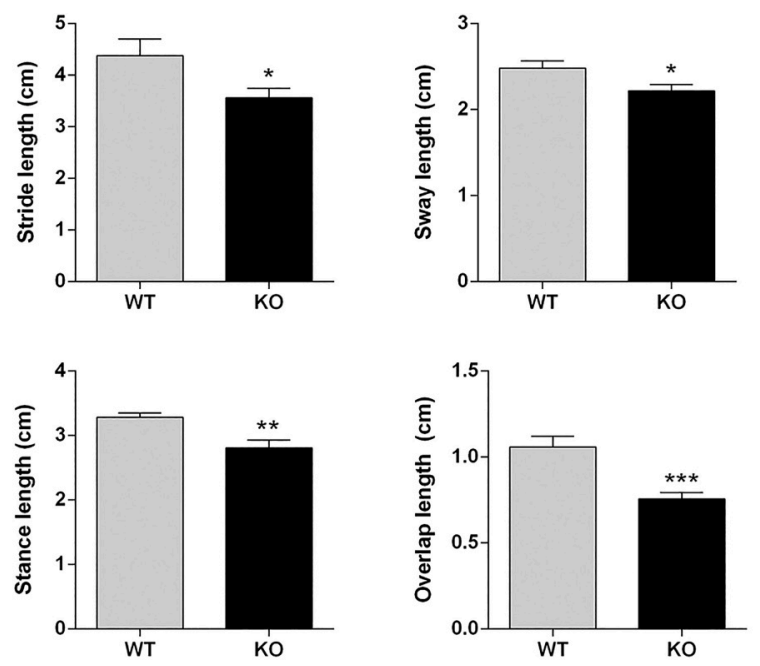
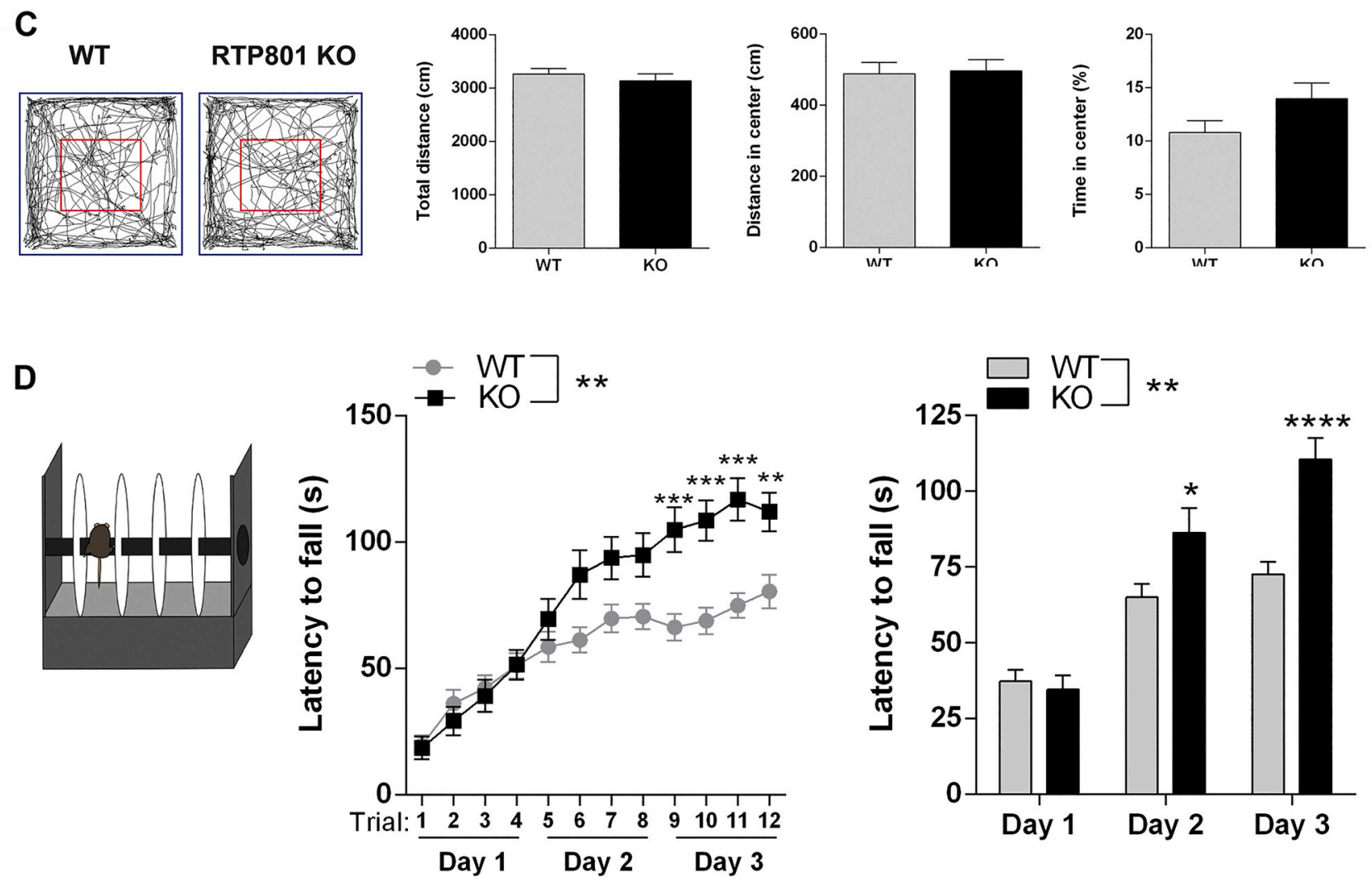

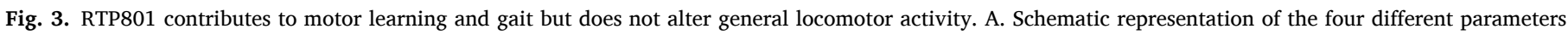

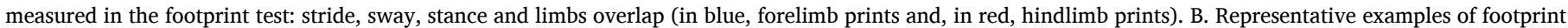

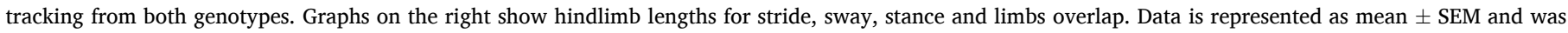

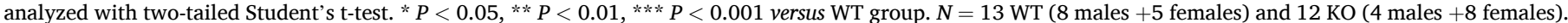

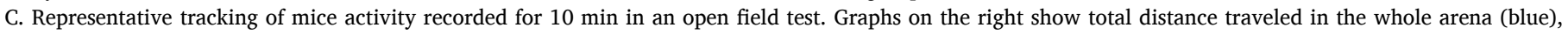

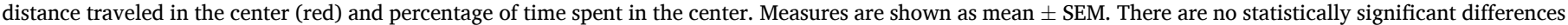

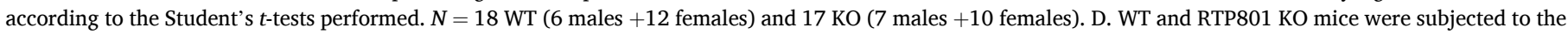

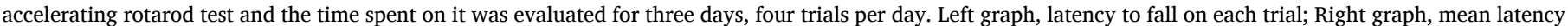

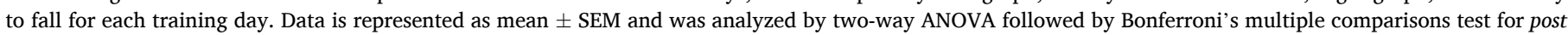

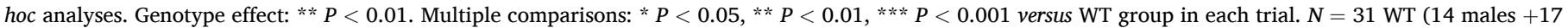
females) and $30 \mathrm{KO}$ (12 males +18 females). 


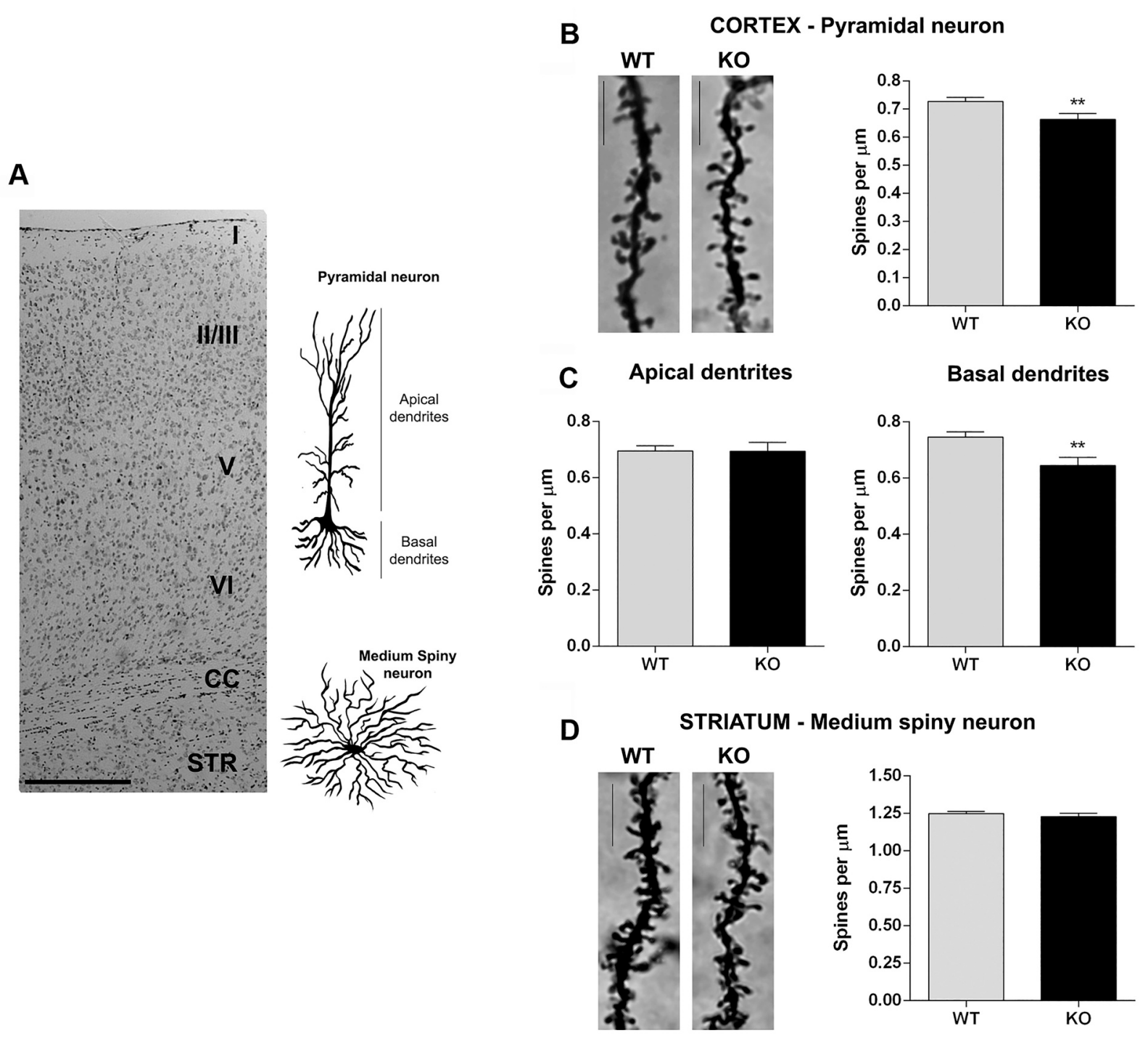

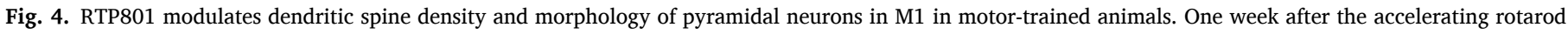

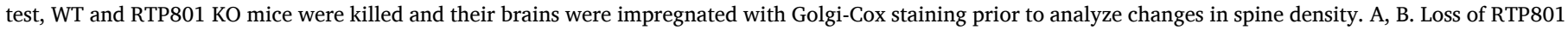

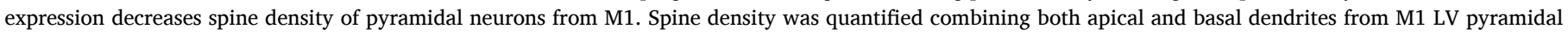

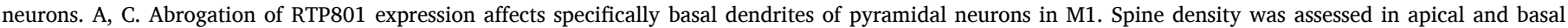

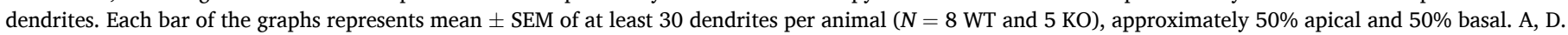

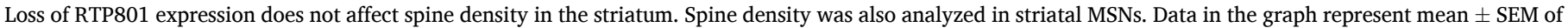

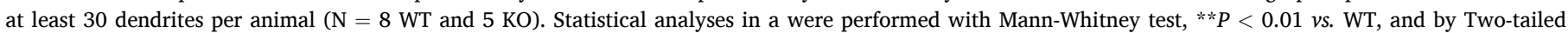

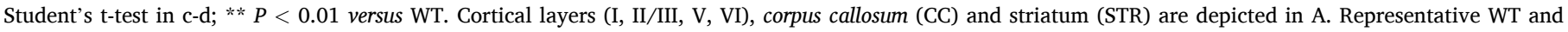
$\mathrm{KO}$ dendrites from primary motor cortex and striatum are shown (B, D). Scale bar, $10 \mu \mathrm{m}$.

learning skills was associated with alterations in dendritic spine structure. Cortical neurons in the motor cortex M1 layer V showed higher number of filopodia- and a mushroom-like morphology and TEM analyses revealed increased postsynaptic and PSD sizes in synaptic contacts from M1 LV. In line with that, trained RTP801 KO mice showed higher levels of synaptic AMPAR subunit GluA1 and a general increase in TrkB levels.

Since the only evidence that RTP801 could modulate synaptic plasticity were found in pathological conditions, here we studied for the first time the putative role of RTP801 in a physiological context. In a context of major depressive disorder, RTP801 KO mice were found resilient to stress-induced synaptic loss in the PFC (Kabir et al., 2017; Ota et al., 2014). Moreover, RTP801 downregulation alleviated stress-induced motor learning dysfunction in a mouse model of genetic PD (Zhang et al., 2018). More recently, our group described that the downregulation of RTP801 restored motor-learning dysfunction in the R6/1 mouse model of HD (Martín-Flores et al., 2020). Hence, the role of downregulation of RTP801 has been proven beneficial in pathological conditions. Our results point out that RTP801 has an important role in spine morphology, neuronal activity and motor behavior in a physiological context.

Hence, we initially confirmed that RTP801 was present in the synapses from a wide range of human and murine samples, as we previously described in HD murine models and HD human postmortem samples (Martín-Flores et al., 2020). Interestingly, RTP801 was highly enriched in human and rat crude synaptosomes but not that elevated in synaptic WT mice samples. In line with that, in cortical cultures RTP801 was localized mostly post-sinaptically. Interestingly, we found that spine density decreased in cortical cultures when RTP801 expression was transiently downregulated and that was translated with an increase in the amplitude and frequency of mEPSCs in KO cortical cultures. An opposite effect was found when ectopic RTP801 was expressed in 
A

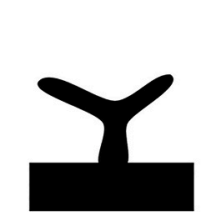

Branched

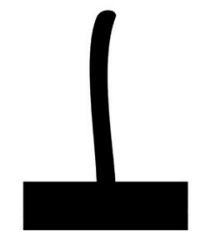

Filopodia

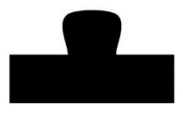

Stubby

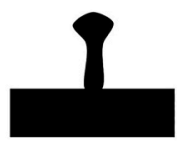

Thin

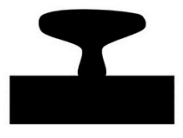

Mushroom
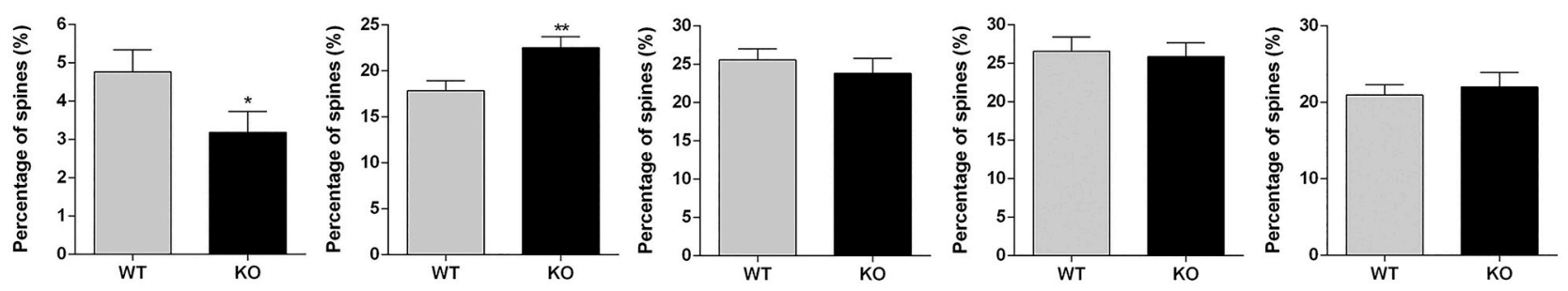

B.1

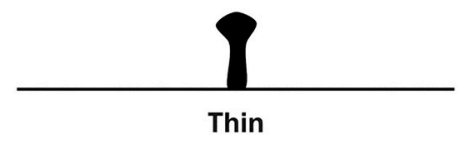

Apical

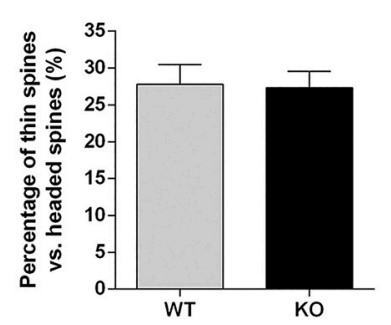

B.2

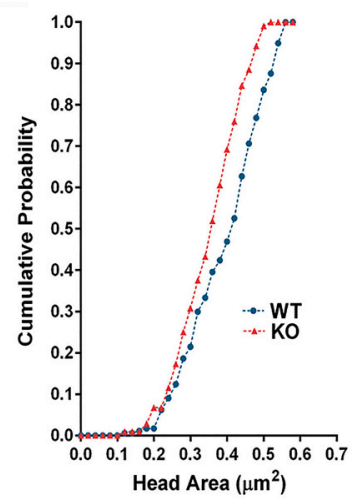

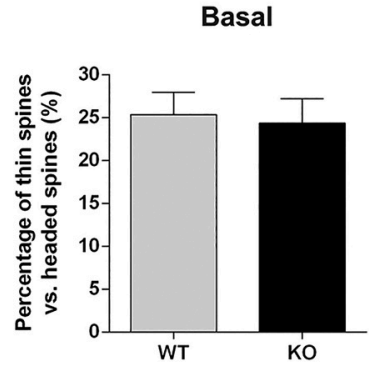

C.2

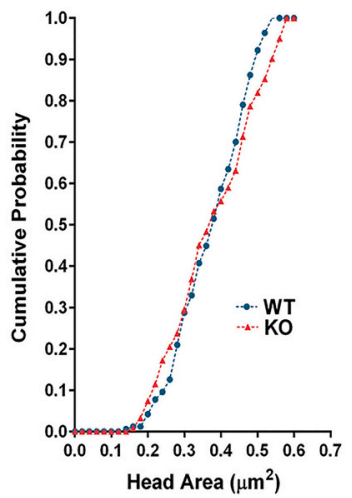

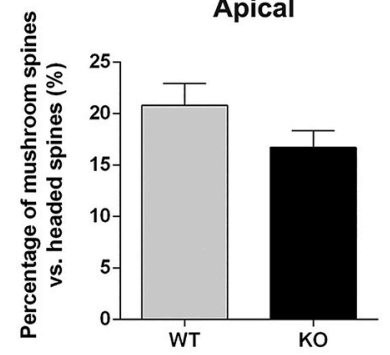

C.1

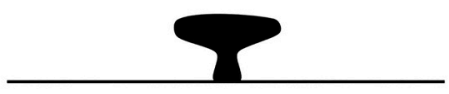

Mushroom

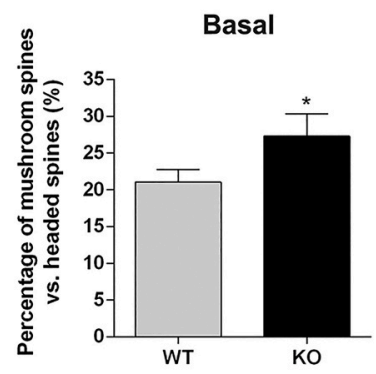

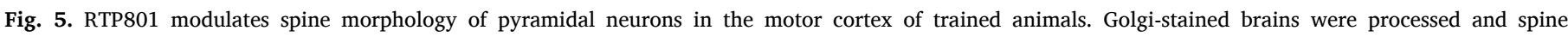

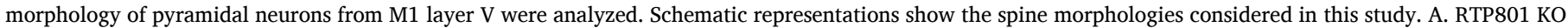

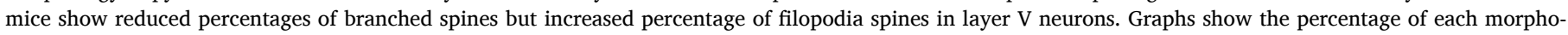

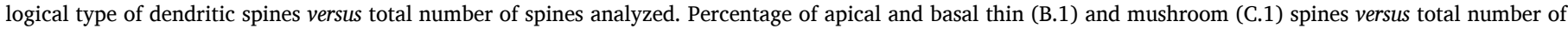

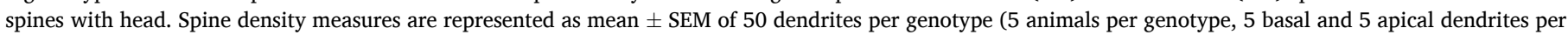

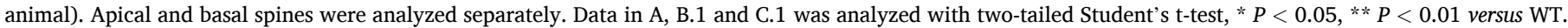

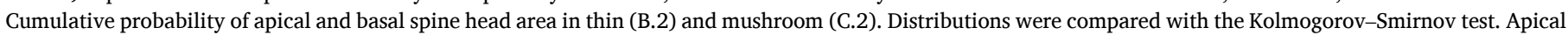

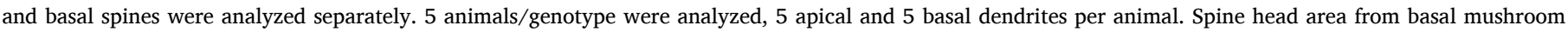
spines shows significant differences between genotypes, $\mathrm{D}=0.2747, P=0.0021$.

hippocampal primary cultures. However, we could not rescue the electrophysiological phenotype in KO neurons because ectopic RTP801 was toxic for them.

Previous studies pointed out that RTP801 KO mice had normal brains and similar behavior to WT animals (Brafman et al., 2004; Ota et al.,
2014). However, no thorough behavioral, biochemical and histological studies were performed in these animals. Macroscopically, we found that the KO mice brain weight less than WT brains, independently of the total body size, and it was likely due to a decrease in the cell density of M1 LV. Noteworthy, this difference can be explained by the 
A
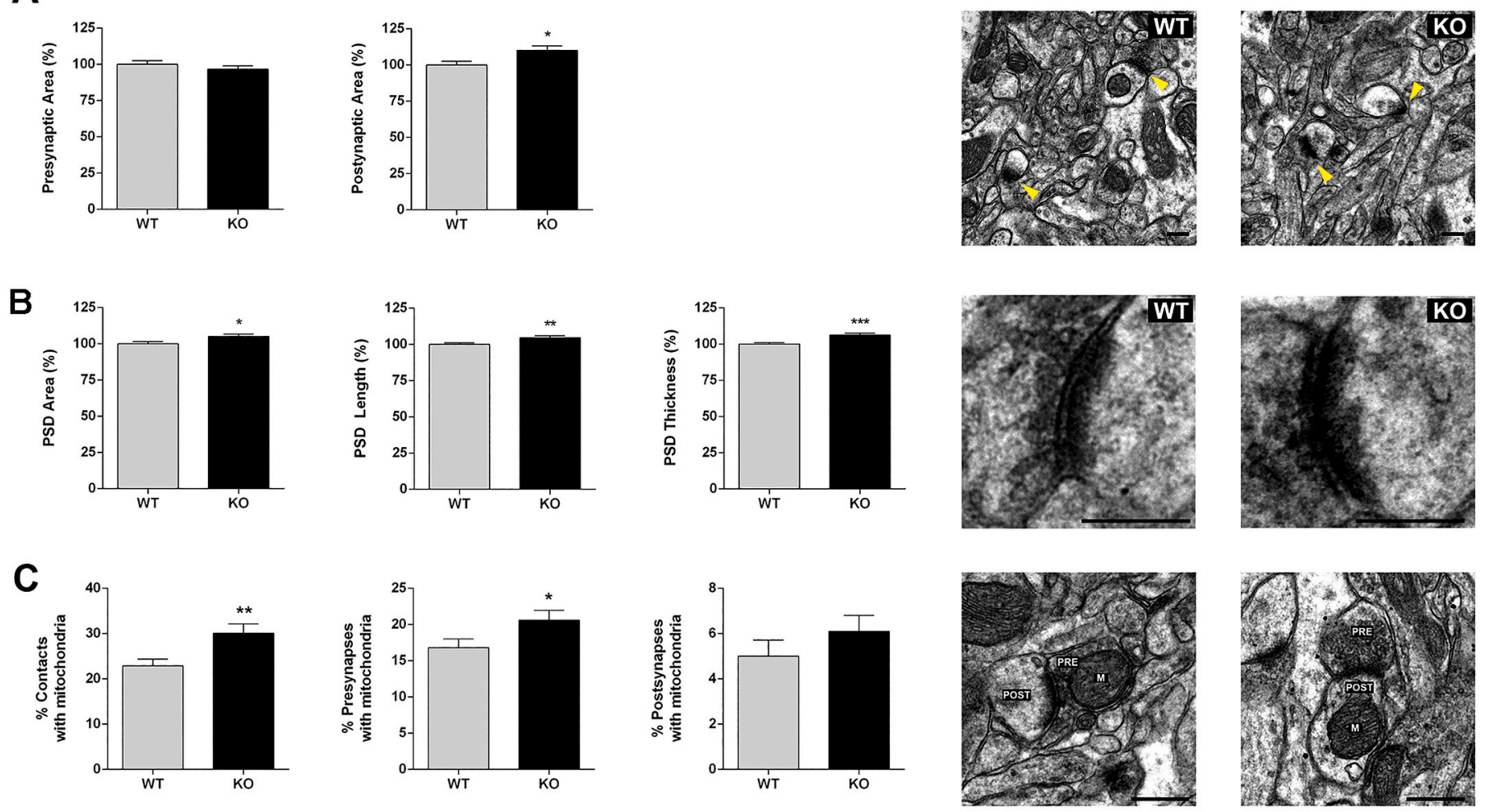

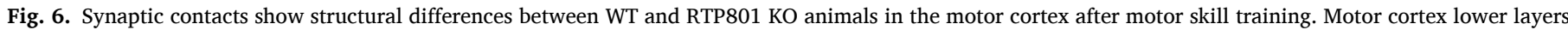

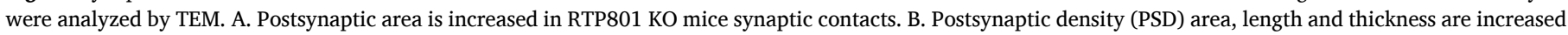

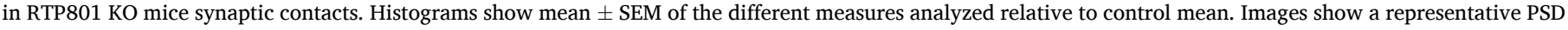

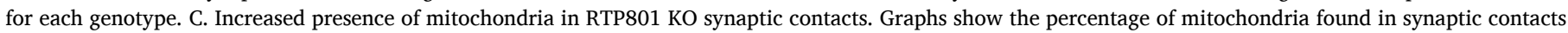

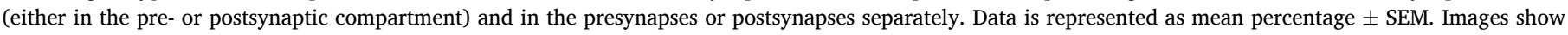

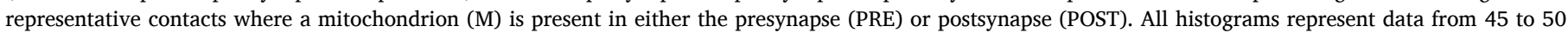

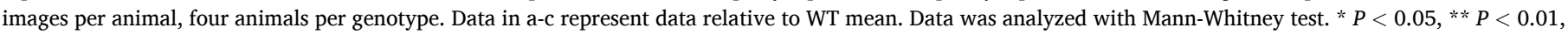
$* * * P<0.001$ versus WT control. For all electron micrographs, Scale bar, $250 \mathrm{~nm}$.

developmental role of RTP801, which regulates both neurogenesis by regulating neuroprogenitors' proliferation rate and neuronal migration/ differentiation in the cortex (Malagelada et al., 2011).

In vivo, the lack of RTP801 reduced spine density in the M1 layer V in the KO mice $v s$. WT. We observed a similar result when we transiently downregulated RTP801 in cultured cortical neurons (Fig. 1C). Interestingly, KO animals showed higher synaptic performance in KO motor cortex (LV) slices versus WT. These results therefore suggest that the lack of RTP801 decreases spine density but enhances synaptic function.

To investigate the role of RTP801 in synaptic plasticity in vivo we performed several motor behavioral tests and checked circuitries that control movement and motor learning. RTP801 KO mice showed gait impairment but no alterations in general locomotor activity. Importantly, we did not observe any epileptic phenotype in the KO mice. It is noteworthy that gait abnormalities are more likely to be explained by cerebellar dysfunction and more studies will be needed in the future. Despite gait alterations, our data show that motor learning does not modulate levels of RTP801 (Fig. S4). Also, spine density in KO mice was reduced compared to WT mice regardless of training (Fig. 2G versus Fig. 4B), suggesting that the levels of RTP801 are relevant for the performance in the task, rather than the task modulating RTP801 levels. More precisely, RTP801 levels might be more relevant for motor learning consolidation, as the initial learning slope is maintained between genotypes, but improved performance is increased from the second day of training. However, our data on HD mice (Martín-Flores et al., 2020) showed that decrease of RTP801 in the striatum of HD mice improved both learning and consolidation of motor learning, suggesting that further investigation is required to elucidate the precise role of
RTP801 in different learning phases.

There is evidence that DDIT4 gene is upregulated in aging brains in animals such as gerbils (Choi et al., 2016) and humans (Elstner et al., 2011), as well as in neurodegenerative conditions such as Parkinson's disease (Malagelada et al., 2006) and Huntington's disease patients (Labadorf et al., 2018; Martin-Flores et al., 2016). Indeed, RTP801/ REDD1 downregulation in the striatum and substantia nigra in the R6/1 mouse model of HD (Martín-Flores et al., 2020) and the A53T model of PD (Zhang et al., 2018), respectively, prevented alterations in motor learning behavior, assessed with the accelerating rotarod test, suggesting a direct role of RTP801 expression levels and motor learning performance, which is in accordance with the present results.

The most characterized circuitry involved in motor learning is the corticostriatal pathway. Pyramidal neurons from the M1 along with striatal MSNs predominantly undergo synaptic dynamics under motor learning (Costa et al., 2004; Tjia et al., 2017). Indeed, spine density in the M1 LV neurons from the RTP801 KO mice, specifically in their basal dendrites, was decreased. We did not observe any differences in spine density in the apical dendrites of the same neurons or in the striatal MSNs from the KO mice. Related to the cortex, Ota and colleagues (Ota et al., 2014) did not find spine density differences in the prefrontal cortex (PFC) between WT and RTP801 KO mice in basal conditions. This fact, together with the absence of differences in the striatum in our work, may point towards a region-specific role of RTP801 in the normal (or physiological, non-stressed) mouse brain. Hence, RTP801 could be contributing to motor learning at the basal dendrites of LV pyramidal neurons. However, the role of RTP801 in other regions involved in motor learning such as cerebellum or brainstem is not yet elucidated. 
A

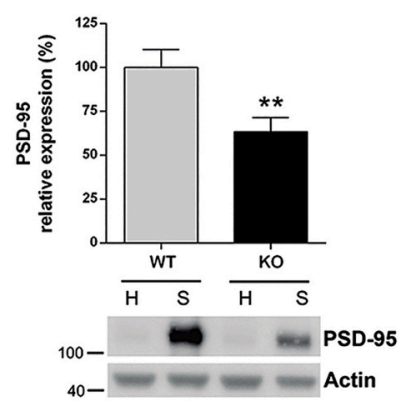

B
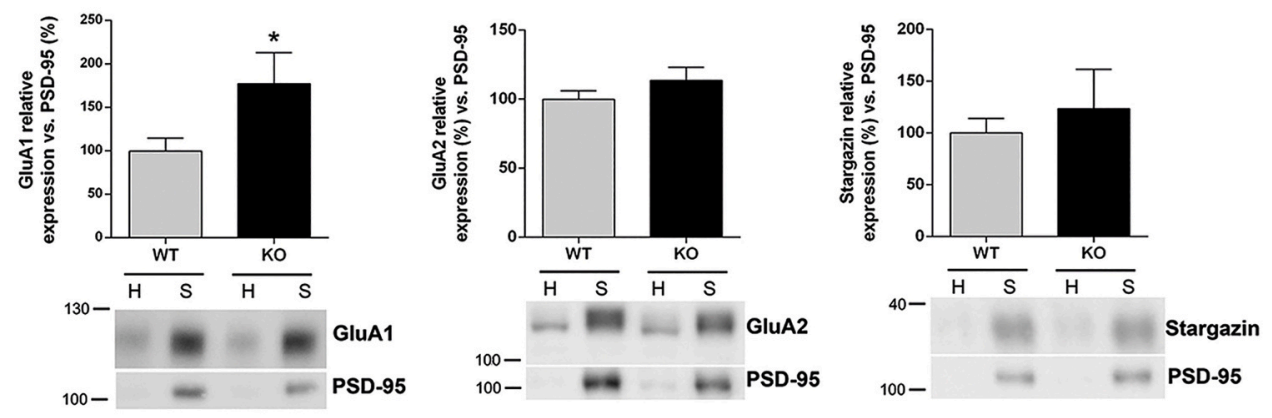

C

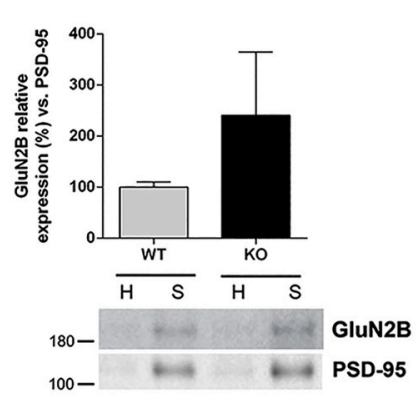

E

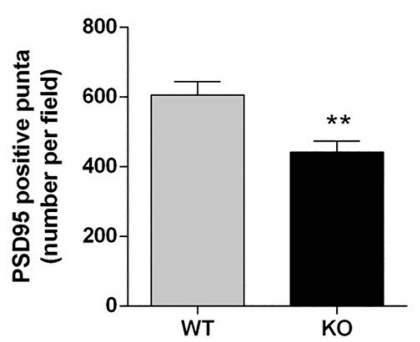

F

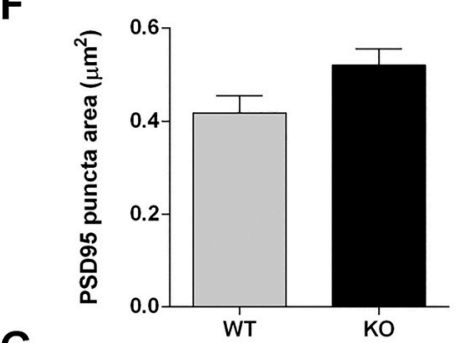

G

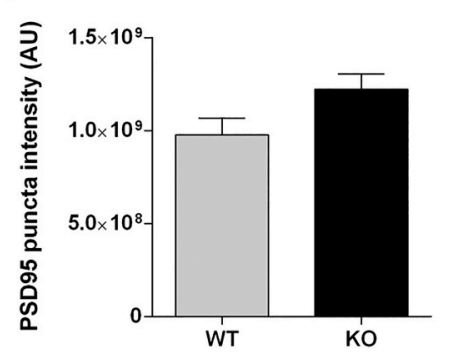

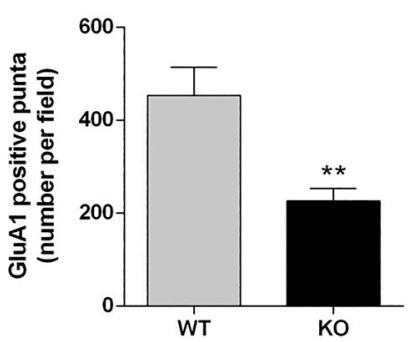
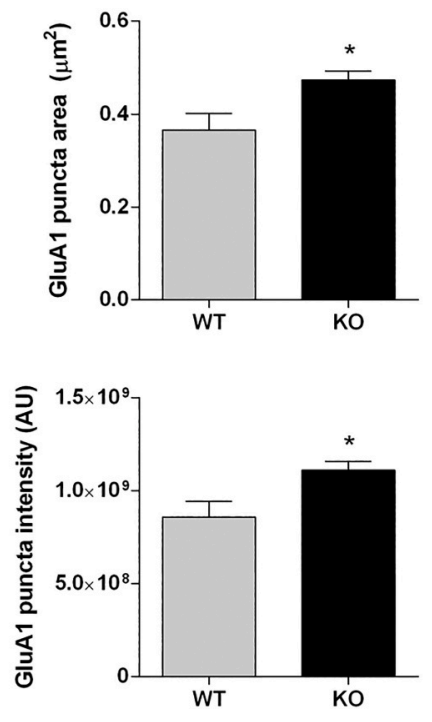

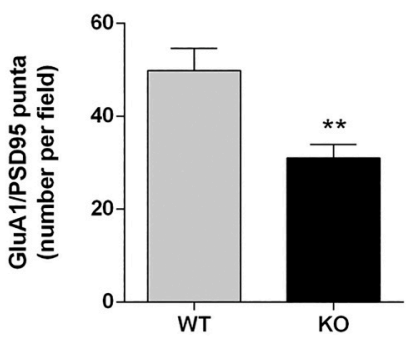

H
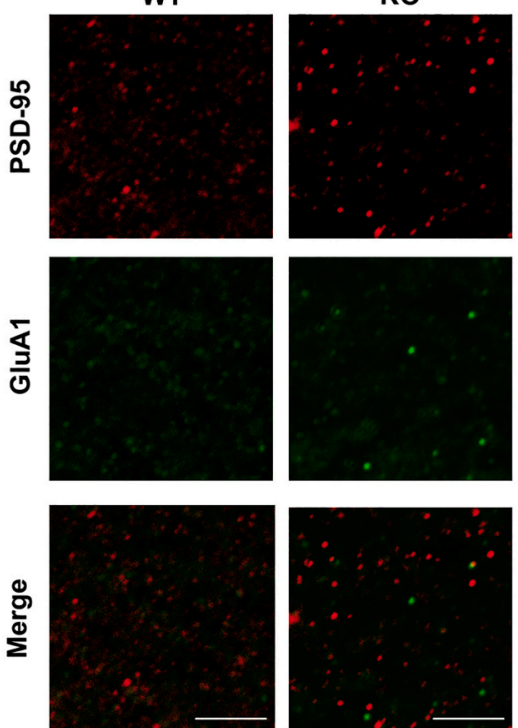

(caption on next page) 


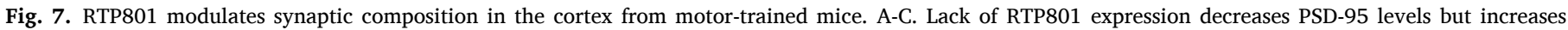

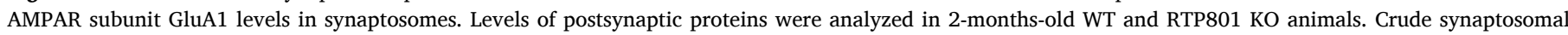

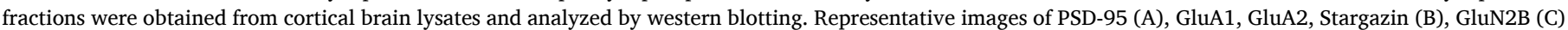

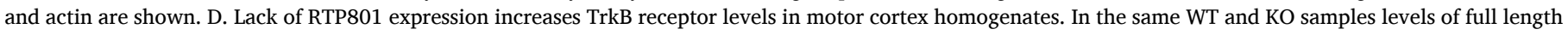

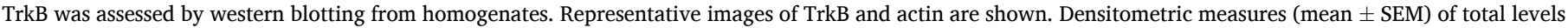

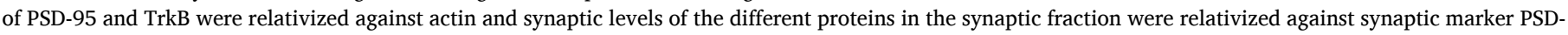

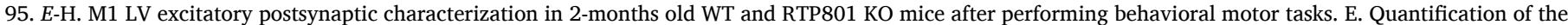

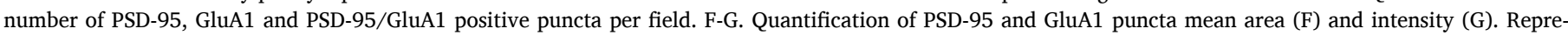

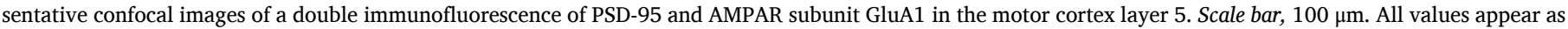
mean \pm SEM and were analyzed with two-tailed Student's t-test versus WT $* P<0.05$ and $* * \mathrm{P}<0.01$

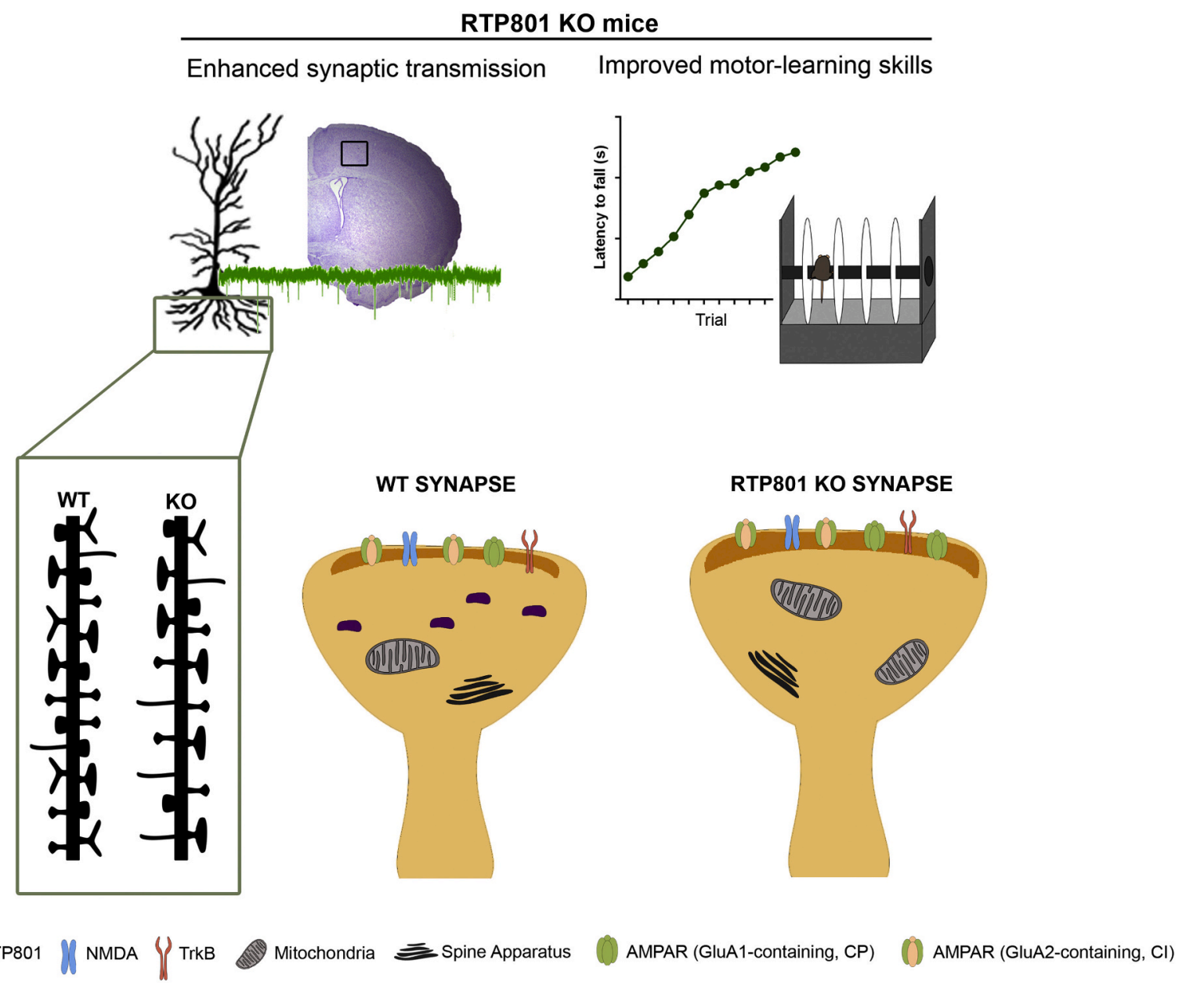

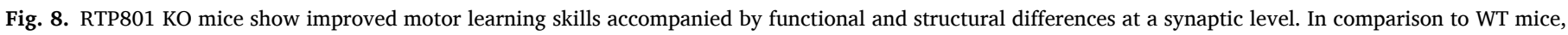

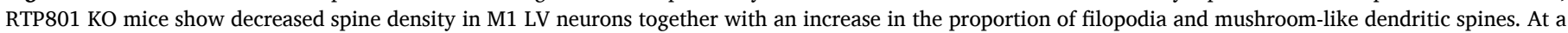

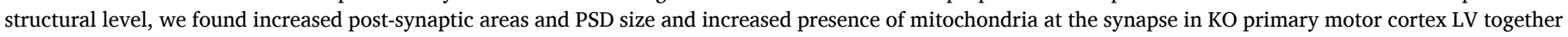
with increased levels of synaptic GluA1 AMPAR subunit.

Precisely, the posterior lobe of the cerebellum is associated with early stages of motor skill learning (Luft and Buitrago, 2005). However as judged by our accelerated rotarod results (see Fig. 3D), learning during the first session is not affected in the KO animals in comparison to WT.

RTP801 modulates the morphology of dendritic spines in M1 LV pyramidal neurons. In this region, we observed a significant increase of filopodia along with a decrease in branched spines in the KO animals. Although the physiological meaning of branched spines is still in debate, filopodia have been proposed to be precursors of spines, to develop an explorative role to increase the probability to form a synapse (Ziv and Smith, 1996; Zuo et al., 2005). However, filopodia-related plasticity must have a fine-tuned regulation, since a high remodeling rate might be troublesome (reviewed in (Ozcan, 2017)). Indeed, among headed spines, we detected an increase in the percentage and head area of mushroom- like spines from basal dendrites between WT and KO animals. This fact correlates well with the change of spine morphology and the function of the spines, and in the end, with an increase in synaptic strength of the area (Arellano et al., 2007; Yuste et al., 2000).

We confirmed a more complex postsynaptic compartment by TEM. The lack of RTP801 led to an increase in postsynaptic area in the contacts of the region of study, although no differences were detected in the presynaptic compartment. Strikingly, greater postsynaptic density size was detected in RTP801 KO animals in the same area. Interestingly, a positive correlation between the amount of PSD and spine size (Arellano et al., 2007) and the former with synaptic strength (Béique and Andrade, 2003; Meyer et al., 2014) has been described. Moreover, KO synaptic contacts present more mitochondria, whose presence at the synapse has been related with a role in controlling plasticity processes (Lee et al., 
2018; Todorova and Blokland, 2017)). Our ultrastructural analyses, therefore, seem to indicate that, although the lack of RTP801 causes a decrease in spine density, the remaining spines are able to compensate this reduction at a structural level.

Interestingly, we observed a differential synaptic composition in the remaining spines in the M1 LV from RTP801 KO mice versus WT animals. We observed decreased levels of synaptic PSD-95 in crude synaptosomes that go in line with the decreased number of PSD-95 positive puncta in M1 LV observed by immunohistochemistry, along with a specific elevation of GluA1 AMPAR subunit at the synapses in M1 LV of KO mice. Calcium impermeable AMPARs (GluA2-containing; CI-AMPARs) are the most prevalent type of AMPAR in neurons (Lu et al., 2009) where they are responsible for postsynaptic currents and the depolarization of the postsynaptic neuron. In contrast, GluA1 subunit confers calcium permeability to the receptor. Calcium permeable AMPAR (CP-AMPARs) are mostly engaged to synaptic regulation and intracellular signaling (reviewed in (Man, 2011). Therefore, the improved performance observed in the KO mice could be explained at least in part with this change in the AMPA receptors subunit composition. This could favor the presence of CP-AMPARs with high calcium permeability and then, in consequence, signaling activation and synaptic regulation. Interestingly, previous studies have demonstrated that motor learning induces an increase in GluA1 levels in dendritic spines in the motor cortex. This increment in GluA1 subunits are key modulators of synaptic plasticity induced by motor skill learning (Roth et al., 2020). The mechanism by which RTP801 could mediate this specific AMPAR subunits composition at the synapses to modulate motor learning has to be explored yet. Ectopic RTP801 overexpression showed the opposite result, since it reduced GluA1 puncta intensity in cultured hippocampal neurons. Remarkably, RTP801 silencing in R6/1 mice induced an increase of total levels of GluA1 and TrkB neurotrophin receptor. Indeed, in trained RTP801 KO mice we could also observe an increase in total levels of TrkB receptor. This result is in line with other works describing that synaptic activity modulates both BDNF levels and TrkB receptors amount and localization (Guo et al., 2014; Lauterborn et al., 2000).

\section{Conclusions}

In summary, our work indicates a novel synaptic function for RTP801 in motor learning and in modulating dendritic spine structure, composition and synaptic plasticity. This finding is important since motor learning alterations and synaptic impairment are key features of neurodegenerative diseases such as PD and HD. Altogether, our results point towards RTP801 downregulation as a promising therapeutic strategy to ameliorate motor learning dysfunction in these diseases.

Supplementary data to this article can be found online at https://doi. org/10.1016/j. expneurol.2021.113755.

\section{Ethical approval and consent to participate}

All procedures were performed in compliance with the NIH Guide for the Care and Use of Laboratory Animals and approved by the local animal care committee of Universitat de Barcelona following European (2010/63/UE) and Spanish (RD53/2013) regulations for the care and use of laboratory animals.

Human samples were obtained following the guidelines and approval of the local ethics committee (Hospital Clínic of Barcelona's Clinical Research Ethics Committee).

\section{Consent for publication}

Not applicable.

\section{Availability of supporting data}

The datasets used and/or analyzed during the current study are available from the corresponding author on reasonable request.

\section{Funding}

Financial support was obtained from the Spanish Ministry of Economy and Competitivity (grants SAF2014-57160-R (AEI/FEDER, UE) for CM and J.A., SAF2017-88076-R (AEI/FEDER, UE) for J.A \& M.J.R., and SAF2017-88812 R (AEI/FEDER, UE) for C.M. We also thank Portal d'Avall S.L. for L.P-S. fellowship. Mice and neuron illustrations were designed by Jorge Padilla Rubio.

\section{Authors' contributions}

L.P-S., N.M-F., M.M., J.S., A.LL., J.R-A., J.S., G.C., M.C., D.S., X.G., J.A and C.M. have contributed to the conception and design of the study, acquisition and analysis of data and in drafting the manuscript and figs. G.C., E.G-G., N.S-F., S.F-G., J.P.G., M.J.R., H-Y.M., E.F. and D.W., have contributed to acquisition and analysis of data and in drafting the manuscript and figures.

\section{Declaration of Competing Interest}

None.

\section{Acknowledgements}

The authors thank Dr. Sílvia Ginés, Dr. Verónica Brito and Dr. Albert Giralt for helpful discussion. We also thank Dr. Albert Martínez from the Faculty of Biology from our same University, for his TEM assessment and guidance. We thank the Neurological Tissue Bank of the BiobancHospital Clínic-IDIBAPS (Barcelona, Spain) and Dr. Ellen Gelpi for providing human tissue samples. We thank Maria Calvo from the Advanced Microscopy Unit, Scientific and Technological Centers, University of Barcelona, for their support and advice in confocal techniques.

\section{References}

Anderson, C.T., Sheets, P.L., Kiritani, T., Shepherd, G.M.G., 2010. Sublayer-specific microcircuits of corticospinal and corticostriatal neurons in motor cortex. Nat. Neurosci. 13, 739-744. https://doi.org/10.1038/nn.2538.

Arellano, J.I., Benavides-Piccione, R., Defelipe, J., Yuste, R., 2007. Ultrastructure of dendritic spines: correlation between synaptic and spine morphologies. Front. Neurosci. 1, 131-143. https://doi.org/10.3389/neuro.01.1.1.010.2007.

Béique, J.C., Andrade, R., 2003. PSD-95 regulates synaptic transmission and plasticity in rat cerebral cortex. J. Physiol. https://doi.org/10.1113/jphysiol.2002.031369.

Bosch, C., Muhaisen, A., Pujadas, L., Soriano, E., Martínez, A., 2016. Reelin exerts structural, biochemical and transcriptional regulation over presynaptic and postsynaptic elements in the adult Hippocampus. Front. Cell. Neurosci. 10, 138. https://doi.org/10.3389/fncel.2016.00138.

Brafman, A., Mett, I., Shafir, M., Gottlieb, H., Damari, G., Gozlan-Kelner, S., VishnevskiaDai, V., Skaliter, R., Einat, P., Faerman, A., Feinstein, E., Shoshani, T., 2004. Inhibition of oxygen-induced retinopathy in RTP801-deficient mice. Invest. Ophthalmol. Vis. Sci. 45, 3796-3805. https://doi.org/10.1167/iovs.04-005245/10/ 3796 (pii).

Calabresi, P., Centonze, D., Gubellini, P., Marfia, G.A., Pisani, A., Sancesario, G., Bernardi, G., 2000. Synaptic transmission in the striatum: from plasticity to neurodegeneration. Prog. Neurobiol. 61, 231-265.

Calabresi, P., Galletti, F., Saggese, E., Ghiglieri, V., Picconi, B., 2007. Neuronal networks and synaptic plasticity in Parkinson's disease: beyond motor deficits. Parkinsonism Relat. Disord. 13 (Suppl. 3), S259-S262. https://doi.org/10.1016/S1353-8020(08) 70013-0.

Canal, M., Martín-Flores, N., Pérez-Sisqués, L., Romaní-Aumedes, J., Altas, B., Man, H.Y., Kawabe, H., Alberch, J., Malagelada, C., 2016. Loss of NEDD4 contributes to RTP801 elevation and neuron toxicity: implications for Parkinson's disease. Oncotarget. https://doi.org/10.18632/oncotarget.11020.

Choi, H.S., Ahn, J.H., Park, J.H., Won, M.H., Lee, C.H., 2016. Age-dependent changes in the protein expression levels of Redd1 and mTOR in the gerbil hippocampus during normal aging. Mol. Med. Rep. 13, 2409-2414. https://doi.org/10.3892/ mmr.2016.4835.

Choi, K., Holly, E.N., Davatolhagh, M.F., Beier, K.T., Fuccillo, M.V., 2019. Integrated anatomical and physiological mapping of striatal afferent projections. Eur. J. Neurosci. 49, 623-636. https://doi.org/10.1111/ejn.13829.

Chou, A.H., Yeh, T.H., Ouyang, P., Chen, Y.L., Chen, S.Y., Wang, H.L., 2008. Polyglutamine-expanded ataxin-3 causes cerebellar dysfunction of SCA3 transgenic 
mice by inducing transcriptional dysregulation. Neurobiol. Dis. 31, 89-101. https:// doi.org/10.1016/j.nbd.2008.03.011.

Costa, R.M., Cohen, D., Nicolelis, M.A.L., 2004. Differential corticostriatal plasticity during fast and slow motor skill learning in mice. Curr. Biol. 14, 1124-1134. https:// doi.org/10.1016/j.cub.2004.06.053.

Creus-Muncunill, J., Rué, L., Alcalá-Vida, R., Badillos-Rodríguez, R., RomaníAumedes, J., Marco, S., Alberch, J., Perez-Otaño, I., Malagelada, C., PérezNavarro, E., 2018. Increased levels of Rictor prevent mutant Huntingtin-induced neuronal degeneration. Mol. Neurobiol. https://doi.org/10.1007/s12035-018-09565.

Elstner, M., Morris, C.M., Heim, K., Bender, A., Mehta, D., Jaros, E., Klopstock, T., Meitinger, T., Turnbull, D.M., Prokisch, H., 2011. Expression analysis of dopaminergic neurons in Parkinson's disease and aging links transcriptional dysregulation of energy metabolism to cell death. Acta Neuropathol. 122, 75-86. https://doi.org/10.1007/s00401-011-0828-9.

Fu, M., Yu, X., Lu, J., Zuo, Y., 2012. Repetitive motor learning induces coordinated formation of clustered dendritic spines in vivo. Nature 483, 92-96. https://doi.org/ 10.1038 /nature10844.

Gilbert, J., Shu, S., Yang, X., Lu, Y., Zhu, L.-Q., Man, H.-Y., 2016. $\beta$-Amyloid triggers aberrant over-scaling of homeostatic synaptic plasticity. Acta Neuropathol. Commun. 4, 131. https://doi.org/10.1186/s40478-016-0398-0.

Guo, W., Ji, Y., Wang, S., Sun, Y., Lu, B., 2014. Neuronal activity alters BDNF-TrkB signaling kinetics and downstream functions. J. Cell Sci. 127, 2249-2260. https:// doi.org/10.1242/jcs.139964.

Guo, L., Xiong, H., Kim, J.-I., Wu, Y.-W., Lalchandani, R.R., Cui, Y., Shu, Y., Xu, T., Ding, J.B., 2015. Dynamic rewiring of neural circuits in the motor cortex in mouse models of Parkinson's disease. Nat. Neurosci. 18, 1299-1309. https://doi.org/ 10.1038/nn.4082.

Guyenet, S.J., Furrer, S.A., Damian, V.M., Baughan, T.D., La Spada, A.R., Garden, G.A., 2010. A simple composite phenotype scoring system for evaluating mouse models of cerebellar ataxia. J. Vis. Exp. https://doi.org/10.3791/1787.

Hintiryan, H., Foster, N.N., Bowman, I., Bay, M., Song, M.Y., Gou, L., Yamashita, S., Bienkowski, M.S., Zingg, B., Zhu, M., Yang, X.W., Shih, J.C., Toga, A.W., Dong, H.W., 2016. The mouse cortico-striatal projectome. Nat. Neurosci. 19, 1100-1114. https:// doi.org/10.1038/nn.4332.

Kabir, Z.D., Lee, A.S., Burgdorf, C.E., Fischer, D.K., Rajadhyaksha, Aditi M., Mok, E., Rizzo, B., Rice, R.C., Singh, K., Ota, K.T., Gerhard, D.M., Schierberl, K.C., Glass, M.J. Duman, R.S., Rajadhyaksha, Anjali M., 2017. Cacna1c in the prefrontal cortex regulates depression-related behaviors via REDD1. Neuropsychopharmacology 42, 2032-2042. https://doi.org/10.1038/npp.2016.271.

Kida, H., Tsuda, Y., Ito, N., Yamamoto, Y., Owada, Y., Kamiya, Y., Mitsushima, D., 2016. Motor training promotes both synaptic and intrinsic plasticity of layer II/III pyramidal neurons in the primary motor cortex. Cereb. Cortex 26, 3494-3507. https://doi.org/10.1093/cercor/bhw134.

Labadorf, A., Choi, S.H., Myers, R.H., 2018. Evidence for a pan-neurodegenerative disease response in Huntington's and Parkinson's disease expression profiles. Front. Mol. Neurosci. 10, 430. https://doi.org/10.3389/fnmol.2017.00430.

Lauterborn, J.C., Lynch, G., Vanderklish, P., Arai, A., Gall, C.M., 2000. Positive modulation of AMPA receptors increases neurotrophin expression by hippocampal and cortical neurons. J. Neurosci. 20, 8-21.

Lee, A., Hirabayashi, Y., Kwon, S.K., Lewis, T.L., Polleux, F., 2018. Emerging roles of mitochondria in synaptic transmission and neurodegeneration. Curr. Opin. Physiol. https://doi.org/10.1016/j.cophys.2018.03.009.

Legendy, C.R., Salcman, M., 1985. Bursts and recurrences of bursts in the spike trains of spontaneously active striate cortex neurons. J. Neurophysiol. 53, 926-939. https:// doi.org/10.1152/jn.1985.53.4.926.

Lu, W., Shi, Y., Jackson, A.C., Bjorgan, K., During, M.J., Sprengel, R., Seeburg, P.H., Nicoll, R.A., 2009. Subunit composition of synaptic AMPA receptors revealed by a single-cell genetic approach. Neuron 62, 254-268. https://doi.org/10.1016/j. neuron.2009.02.027.

Luft, A.R., Buitrago, M.M., 2005. Stages of motor skill learning. Mol. Neurobiol. https:// doi.org/10.1385/MN:32:3:205.

Malagelada, C., Ryu, E.J., Biswas, S.C., Jackson-Lewis, V., Greene, L.A., 2006. RTP801 is elevated in Parkinson brain substantia nigral neurons and mediates death in cellular models of Parkinson's disease by a mechanism involving mammalian target of rapamycin inactivation. J. Neurosci. 26, 9996-10005. https://doi.org/10.1523/ JNEUROSCI.3292-06.2006.

Malagelada, C., Zong, H.J., Greene, L.A., 2008. RTP801 is induced in Parkinson's disease and mediates neuron death by inhibiting Akt phosphorylation/activation. J. Neurosci. 28 https://doi.org/10.1523/JNEUROSCI.3928-08.2008.

Malagelada, C., Lopez-Toledano, M.A., Willett, R.T., Jin, Z.H., Shelanski, M.L., Greene, L. A., 2011. RTP801/REDD1 regulates the timing of cortical neurogenesis and neuron migration. J. Neurosci. 31, 3186-3196. https://doi.org/10.1523/JNEUROSCI.401110.2011 .

Man, H.-Y., 2011. GluA2-lacking, calcium-permeable AMPA receptors — inducers of plasticity? Curr. Opin. Neurobiol. 21, 291-298. https://doi.org/10.1016/j. conb.2011.01.001.

Mangiarini, L., Sathasivam, K., Seller, M., Cozens, B., Harper, A., Hetherington, C., Lawton, M., Trottier, Y., Lehrach, H., Davies, S.W., Bates, G.P., 1996. Exon I of the HD gene with an expanded CAG repeat is sufficient to cause a progressive neurological phenotype in transgenic mice. Cell 87, 493-506. https://doi.org/ 10.1016/S0092-8674(00)81369-0.

Martín-Flores, N., Romaní-Aumedes, J., Rué, L., Canal, M., Sanders, P., Straccia, M., Allen, N.D., Alberch, J., Canals, J.M., Pérez-Navarro, E., Malagelada, C., 2016. RTP801 is involved in mutant Huntingtin-induced cell death. Mol. Neurobiol. 53, 2857-2868. https://doi.org/10.1007/s12035-015-9166-6.

Martín-Flores, N., Pérez-Sisqués, L., Creus-Muncunill, J., Masana, M., Ginés, S., Alberch, J., Pérez-Navarro, E., Malagelada, C., 2020. Synaptic RTP801 contributes to motor-learning dysfunction in Huntington's disease. Cell Death Dis. 11, 1-15. https://doi.org/10.1038/s41419-020-02775-5.

McIntire, S.L., Reimer, R.J., Schuske, K., Edwards, R.H., Jorgensen, E.M., 1997. Identification and characterization of the vesicular GABA transporter. Nature 389 , 870-876. https://doi.org/10.1038/39908.

Meyer, D., Bonhoeffer, T., Scheuss, V., 2014. Balance and stability of synaptic structures during synaptic plasticity. Neuron 82, 430-443. https://doi.org/10.1016/j. neuron.2014.02.031.

Noseda, R., Belin, S., Piguet, F., Vaccari, I., Scarlino, S., Brambilla, P., Martinelli Boneschi, F., Feltri, M.L., Wrabetz, L., Quattrini, A., Feinstein, E., Huganir, R.L., Bolino, A., 2013. DDIT4/REDD1/RTP801 is a novel negative regulator of Schwann cell myelination. J. Neurosci. 33, 15295-15305. https://doi.org/10.1523/ JNEUROSCI.2408-13.2013.

Ota, K.T., Liu, R.J., Voleti, B., Maldonado-Aviles, J.G., Duric, V., Iwata, M., Dutheil, S., Duman, C., Boikess, S., Lewis, D.A., Stockmeier, C.A., DiLeone, R.J., Rex, C., Aghajanian, G.K., Duman, R.S., 2014. REDD1 is essential for stress-induced synaptic loss and depressive behavior. Nat. Med. 20, 531-535. https://doi.org/10.1038/ nm.3513.

Ozcan, A.S., 2017. Filopodia: a rapid structural plasticity substrate for fast learning. Front. Synaptic Neurosci. 9, 12. https://doi.org/10.3389/fnsyn.2017.00012.

Peters, A.J., Liu, H., Komiyama, T., 2017. Learning in the rodent motor cortex. Annu. Rev. Neurosci. 40, 77-97. https://doi.org/10.1146/annurev-neuro-072116-031407.

Romaní-Aumedes, J., Canal, M., Martín-Flores, N., Sun, X., Pérez-Fernández, V., Wewering, S., Fernández-Santiago, R., Ezquerra, M., Pont-Sunyer, C., Lafuente, A., Alberch, J., Luebbert, H., Tolosa, E., Levy, O.A., Greene, L.A., Malagelada, C., 2014. Parkin loss of function contributes to RTP801 elevation and neurodegeneration in Parkinson's disease. Cell Death Dis. 5 https://doi.org/10.1038/cddis.2014.333.

Roth, R.H., Cudmore, R.H., Tan, H.L., Hong, I., Zhang, Y., Huganir, R.L., 2020. Cortical synaptic AMPA receptor plasticity during motor learning. Neuron 105, 895-908 e5. https://doi.org/10.1016/j.neuron.2019.12.005.

Rothman, J.S., Silver, R.A., 2018. NeuroMatic: an integrated open-source software toolkit for acquisition, analysis and simulation of electrophysiological data. Front. Neuroinform. 12 https://doi.org/10.3389/fninf.2018.00014.

Ryu, E.J., Angelastro, J.M., Greene, L.A., 2005. Analysis of gene expression changes in a cellular model of Parkinson disease. Neurobiol. Dis. 18, 54-74 doi: S0969-9961(04) 00211-6 [pii]. https://doi.org/10.1016/j.nbd.2004.08.016.

Sanes, J.N., Donoghue, J.P., 2000. Plasticity and primary motor cortex. Annu. Rev. Neurosci. 23, 393-415. https://doi.org/10.1146/annurev.neuro.23.1.393.

Shepherd, G.M.G., 2013. Corticostriatal connectivity and its role in disease. Nat. Rev. Neurosci. https://doi.org/10.1038/nrn3469.

Shepherd, J.D., Huganir, R.L., 2007. The cell biology of synaptic plasticity: AMPA receptor trafficking. Annu. Rev. Cell Dev. Biol. 23, 613-643. https://doi.org/ 10.1146/annurev.cellbio.23.090506.123516.

Shoshani, T., Faerman, A., Mett, I., Zelin, E., Tenne, T., Gorodin, S., Moshel, Y., Elbaz, S., Budanov, A., Chajut, A., Kalinski, H., Kamer, I., Rozen, A., Mor, O., Keshet, E., Leshkowitz, D., Einat, P., Skaliter, R., Feinstein, E., 2002. Identification of a novel hypoxia-inducible factor 1-responsive gene, RTP801, involved in apoptosis. Mol. Cell. Biol. 22, 2283-2293.

Tjia, M., Yu, X., Jammu, L.S., Lu, J., Zuo, Y., 2017. Pyramidal neurons in different cortical layers exhibit distinct dynamics and plasticity of apical dendritic spines. Front. Neural Circuits 11, 43. https://doi.org/10.3389/fncir.2017.00043.

Todorova, V., Blokland, A., 2017. Mitochondria and synaptic plasticity in the mature and aging nervous system. Curr. Neuropharmacol. 15, 166-173.

Xu, T., Yu, X., Perlik, A.J., Tobin, W.F., Zweig, J.A., Tennant, K., Jones, T., Zuo, Y., 2009. Rapid formation and selective stabilization of synapses for enduring motor memories. Nature 462, 915-919. https://doi.org/10.1038/nature08389.

Xu, T., Wang, S., Lalchandani, R.R., Ding, J.B., 2017. Motor learning in animal models of Parkinson's disease: aberrant synaptic plasticity in the motor cortex. Mov. Disord. 32, 487-497. https://doi.org/10.1002/mds.26938.

Yuste, R., Majewska, A., Holthoff, K., 2000. From form to function: calcium compartmentalization in dendritic spines. Nat. Neurosci. 3, 653-659. https://doi. org/10.1038/76609.

Zhang, Z., Chu, S.-F., Wang, S.-S., Jiang, Y.-N., Gao, Y., Yang, P.-F., Ai, Q.-D., Chen, N.H., 2018. RTP801 is a critical factor in the neurodegeneration process of A53T $\alpha$-synuclein in a mouse model of Parkinson's disease under chronic restraint stress. Br. J. Pharmacol. 175, 590-605. https://doi.org/10.1111/bph.14091.

Ziv, N.E., Smith, S.J., 1996. Evidence for a role of dendritic filopodia in synaptogenesis and spine formation. Neuron 17, 91-102. https://doi.org/10.1016/S0896-6273(00) 80283-4.

Zuo, Y., Lin, A., Chang, P., Gan, W.-B., 2005. Development of long-term dendritic spine stability in diverse regions of cerebral cortex. Neuron 46, 181-189. https://doi.org/ 10.1016/J.NEURON.2005.04.001. 